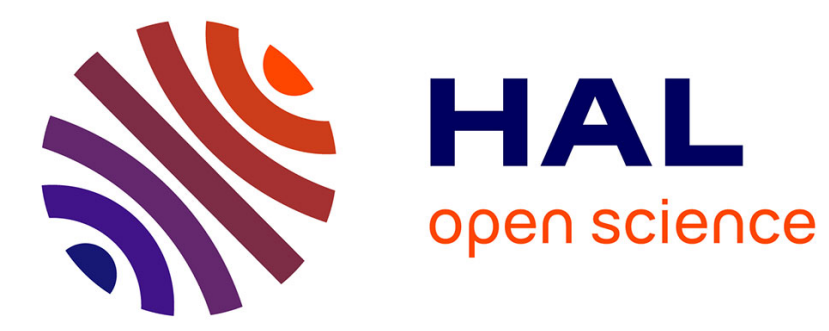

\title{
Résistivité électrique à basse température du niobium et d'alliages niobium-hafnium en phase cubique
}

\author{
P. Haen, J. Teixeira
}

\section{To cite this version:}

P. Haen, J. Teixeira. Résistivité électrique à basse température du niobium et d'alliages niobium-hafnium en phase cubique. Revue de Physique Appliquée, 1974, 9 (5), pp.879-893. 10.1051/rphysap:0197400905087900 . jpa-00243853

HAL Id: jpa-00243853

https://hal.science/jpa-00243853

Submitted on 1 Jan 1974

HAL is a multi-disciplinary open access archive for the deposit and dissemination of scientific research documents, whether they are published or not. The documents may come from teaching and research institutions in France or abroad, or from public or private research centers.
L'archive ouverte pluridisciplinaire HAL, est destinée au dépôt et à la diffusion de documents scientifiques de niveau recherche, publiés ou non, émanant des établissements d'enseignement et de recherche français ou étrangers, des laboratoires publics ou privés. 


\title{
RÉSISTIVITÉ ÉLECTRIQUE A BASSE TEMPÉRATURE DU NIOBIUM ET D'AlLIAGES NIOBIUM-HAFNIUM EN PHASE CUBIQUE
}

\author{
P. HAEN et J. TEIXEIRA \\ Centre de Recherches sur Les Très Basses Températures, C. N. R. S., \\ B. P. 166, 38042 Grenoble Cedex, France
}

(Reçu le 16 juillet 1973, révisé le 20 février 1974)

Résumé. - Nous avons mesuré, entre leur température de transition supraconductrice et l'ambiante, la résistivité électrique de deux échantillons de niobium et d'échantillons d'alliages $\mathrm{Nb}-\mathrm{Hf}$ en phase cubique, de concentration comprise entre 6 et $56 \%$ at. Hf. L'appareillage utilisé permet également la mesure de la largeur de transitions supraconductrices. En traitant numériquement les résultats, nous avons déterminé une expression mathématique de la résistivité des échantillons. Pour le niobium, cette expression est analogue à celle précédemment obtenue par Webb, c'est-à-dire de la forme

$$
\rho=A+B T^{2}+C\left(\frac{T}{\theta}\right)^{3} J_{3}\left(\frac{\theta}{T}\right)+D\left(\frac{T}{\theta}\right)^{5} J_{5}\left(\frac{\theta}{T}\right)
$$

où $J_{3}(\theta / T)$ et $J_{5}(\theta / T)$ sont des intégrales du type

$$
J_{n}(x)=\int_{0}^{x} z^{n} e^{z}\left(e^{z}-1\right)^{-2} \mathrm{~d} z
$$

Dans le niobium, la contribution du terme $J_{5}(\theta / T)$ diminue quand le rapport de résistivité décroît. Cela est en accord avec l'existence d'écarts à la règle de Matthiessen d'autant plus grands que ce rapport est plus faible. Dans les alliages Nb-Hf le terme en $J_{5}$ est nul et les écarts varient en $T^{3}$ à basse température. L'évolution des paramètres $B, C$ et $\theta$ avec la concentration est discutée, et, dans le cas de $\theta$, comparée à celle obtenue par mesure de chaleur spécifique sur des alliages du même type (Nb-Zr, Nb-Ti, Ta-Hf).

Abstract. - We have measured the electrical resistivity of two samples of niobium and of bcc $\mathrm{Nb}-\mathrm{Hf}$ alloys with various hafnium concentrations (6 to 56 at. \%) between their superconducting transition temperatures and room temperature. The apparatus used is also capable of measuring the width of superconducting transitions. By numerical analysis of the results, we have determined a mathematical expression for the resistivity of our specimens. For niobium, this expression is similar to that obtained by Webb and has the form :

$$
\rho=A+B T^{2}+C\left(\frac{T}{\theta}\right)^{3} J_{3}\left(\frac{\theta}{T}\right)+D\left(\frac{T}{\theta}\right)^{5} J_{5}\left(\frac{\theta}{T}\right)
$$

where $J_{3}(\theta / T)$ and $J_{5}(\theta / T)$ are integrals of the type

$$
J_{n}(x)=\int_{0}^{x} z^{n} e^{z}\left(e^{z}-1\right)^{-2} \mathrm{~d} z
$$

For niobium, the contribution of the $J_{5}(\theta / T)$ term diminishes when the residual resistivity ratio decreases. This agrees with the existence of deviations from Matthiessen's rule which become larger for lower values of this ratio. For the Nb-Hf alloys, the $J_{5}$ term is zero and the deviations have a $T^{3}$ variation at low temperatures. The evolution with concentration of the parameters $B$, $C$ and $\theta$ is discussed, and, in the case of $\theta$, compared to the values obtained by specific heat measurements on alloys of the same kind ( $\mathrm{Nb}-\mathrm{Zr}, \mathrm{Nb}-\mathrm{Ti}, \mathrm{Ta}-\mathrm{Hf}$ ).

1. Introduction. - La résistivité électrique des alliages constitués de zirconium ou de titane et d'un métal à structure cubique centrée du groupe $\mathrm{V}, \mathrm{Nb}, \mathrm{Ta}$ ou des groupes suivants, présente un certain nombre de propriétés intéressantes, telles que les variations liées aux transformations à l'état solide de la phase cubique centrée $\beta$ dans les alliages riches en $\mathrm{Ti}$ ou $\mathrm{Zr}$ [1]. On peut supposer que la résistivité des alliages du même type à base de hafnium possède également des propriétés particulières mais il semble qu'aucune étude n'ait encore été faite à ce sujet. Pour ce qui est des alliages $\mathrm{Nb}-\mathrm{Hf}$, on ne disposait jusqu'à présent, dans 
le domaine des basses températures, que de mesures de résistivité résiduelle et de température critique, effectuées généralement en annexe à des investigations sur leurs propriétés métallurgiques ou supraconductrices [2-7]. C'est pourquoi, à la suite des travaux menés dans ce laboratoire par Béthoux et Guérin sur la supraconductivité de ces alliages [5, 6], nous avons abordé l'étude de leur résistivité électrique, et en premier lieu celle des alliages en phase $\beta$, dont nous présentons ici les résultats. Dans ce but, nous avons sélectionné, parmi les échantillons de Béthoux et Guérin, ceux de teneur inférieure à $56 \%$ atomiques de Hf, préparés pour avoir la structure $\beta$. Nous avons mesuré leur température critique et leur résistivité électrique entre $T_{\mathrm{c}}$ et l'ambiante, et également, à titre complémentaire, celles de deux échantillons de niobium.

Nous avons traité nos résultats de résistivité électrique d'une manière analogue à celle utilisée par Webb dans le cas du niobium [3]. Cela consiste à déterminer, à partir des points expérimentaux, par une méthode de moindres carrés, les paramètres d'une loi basée sur les théories classiques de la résistivité. On sait qu'un tel calcul fournit une valeur de la température de Debye, mais il permet également de mettre en évidence les différentes contributions à la résistivité. Nous avons ainsi obtenu pour le niobium des résultats en accord avec ceux de Webb, bien que nos échantillons aient été d'une pureté nettement moindre.

Nous ne possédons pas d'exemples où cette méthode ait été appliquée à des alliages, et en particulier à des alliages concentrés. Il faut pour cela adapter à ceux-ci les lois de variation de la résistivité établies pour des métaux purs. Accessoirement, si la résistivité résiduelle devient importante, comme c'est le cas de nos alliages les plus concentrés, on risque de ne plus pouvoir déterminer les autres contributions avec une précision suffisante. Mais on verra que pour ce qui est des alliages $\mathrm{Nb}-\mathrm{Hf}$, ce traitement des résultats fournit des renseignements intéressants, tant en ce qui concerne l'évolution avec la concentration des différentes contributions à la résistivité électrique, que la variation de la température de Debye, qui peuvent se comparer utilement à ceux que l'on obtient par mesure de chaleur spécifique. Enfin, les expressions analytiques de la résistivité ainsi établies seront utilisées directement pour calculer les écarts à la règle de Matthiessen.

2. Elaboration et méthode de mesure des échantillons. - 2.1 ElABORATION DES ÉCHANTILlONS. - Les échantillons de niobium ont été obtenus à partir de métal, refondu par bombardement électronique, fourni par Ugine-Kuhlmann et qui contenait moins de 250 ppm d'impuretés métalliques. L'échantillon $n^{\circ} 1$ destiné à être mesuré dans les mêmes conditions que les alliages, avait comme ceux-ci un diamètre de $3 \mathrm{~mm}$ et environ $20 \mathrm{~mm}$ de long ; il provenait d'un barreau qui, brut de fusion, avait $5 \mathrm{~mm}$ de diamètre et n'a pas été recuit. Les échantillons $n^{\circ} 2$ et $n^{\circ} 3$ étaient des fils de $0,9 \mathrm{~mm}$ de diamètre et $8 \mathrm{~cm}$ de long, recuits sous ultravide. Nous les nommerons $\mathrm{Nb} 1, \mathrm{Nb} 2$ et $\mathrm{Nb} 3$.

Les alliages ont été élaborés dans les laboratoires de la Société Ugine-Kuhlmann. Le niobium utilisé contenait, suivant sa provenance, de 500 à $3000 \mathrm{ppm}$ de tantale, et l'hafnium $0,63 \%$ de zirconium. Dans chacun de ces deux constituants le total des autres impuretés métalliques était voisin de $250 \mathrm{ppm}$. La fusion avait lieu au four à bombardement électronique sous un vide de $10^{-4}$ torr ; chaque lingot d'alliage résultait de plusieurs fusions successives. Par forgeage à $1000{ }^{\circ} \mathrm{C}$ puis usinage, on en tirait un rondin de $8,5 \mathrm{~mm}$ de diamètre, lequel était ensuite martelé à froid jusqu'à un diamètre de $3 \mathrm{~mm}$. Dans les barreaux ainsi obtenus étaient découpés des échantillons d'environ $20 \mathrm{~mm}$ de long.

La teneur en hafnium de chaque lingot a été déterminée par fluorescence de rayons $X$. La quantité d'impuretés métalliques n'a pas été analysée ; on peut admettre qu'elle était proportionnelle à celle des métaux de départ. On a, par contre, dosé le carbone, l'oxygène et l'azote dont la teneur était de 40 à 50 ppm pour les deux premiers et 15 à 20 ppm pour le dernier.

La liste des échantillons étudiés est donnée dans le tableau I. Nous avons généralement mesuré deux échantillons issus du même barreau, l'un brut de martelage, l'autre recuit sous vide pendant $3 \mathrm{~h}$ à $1000^{\circ} \mathrm{C}$ et trempé à l'huile. Pour les concentrations de 27,2 et $27,4 \%$, c'est même au total 5 échantillons que nous avons mesurés afin de tester la reproductibilité des résultats. En outre, pour certaines concentrations, nous donnons $T_{\mathrm{c}}, \rho_{0}$ et $\rho(300) / \rho_{0}$ d'échantillons de $0,6 \mathrm{~mm}$ de diamètre, obtenus par tréfilage d'une partie d'un barreau de $3 \mathrm{~mm}$. Les lettres $E$ et $R$ désignent respectivement l'état écroui et recuit.

L'élaboration de ces échantillons avait été accompagnée d'une étude du diagramme niobium-hafnium qui, par métallographie et diffraction de rayons $\mathrm{X}$, montrait que les alliages préparés dans les conditions énoncées ci-dessus, étaient, tant à l'état brut de fusion que martelés ou recuits, en phase cubique centrée jusqu'à $65 \%$ atomique de hafnium. Pour cette concentration, cela situait la limite entre les domaines $\beta$ et $\alpha+\beta$ à une température comprise entre 900 et $1000^{\circ} \mathrm{C}$, c'est-à-dire beaucoup plus bas que celle qui était donnée par ailleurs [9-11]. Plus récemment, Carpenter et al. $[12,7]$ ont réétudié cette limite et la situent à $1200^{\circ} \mathrm{C}$ pour un alliage à $38 \%$ at. Hf. C'est par microscopie électronique qu'ils ont montré qu'un recuit au-dessous de cette température provoque la formation de zones, puis de précipités partiellement cohérents de phase $\alpha$. Il n'est pas impossible qu'un tel phénomène se soit produit dans nos échantillons les plus concentrés au cours de leur élaboration, mais il n'a pas été décelé sur les microscopies électroniques que nous avons effectuées ; celles-ci montraient principalement les effets de l'écrouissage, lesquels subsistent partiellement dans les échantillons recuits. 


\begin{tabular}{|c|c|c|c|c|c|c|c|c|c|c|}
\hline & & & & & BLEAU & & & & & \\
\hline & & & $T_{\mathrm{c}}$ & $\rho_{0}$ & $\underline{\rho(300)}$ & $\theta$ & $A$ & $B$ & $C$ & $\sigma$ \\
\hline $\begin{array}{l}\text { Teneur en } \mathrm{Hf} \\
(\% \text { at. })\end{array}$ & $\begin{array}{l}\text { Diamètre } \\
(\mathrm{mm})\end{array}$ & $\begin{array}{l}\text { métallur- } \\
\text { gique }\end{array}$ & $(\mathrm{K})$ & $(\mu \Omega . \mathrm{cm})$ & $\rho_{0}$ & $(\mathrm{~K})$ & $(\mu \Omega . \mathrm{cm})$ & $\underset{\left(\times 10^{5}\right)}{\left(\mu \Omega \cdot c m \cdot K^{-2}\right)}$ & $(\mu \Omega . \mathrm{cm})$ & $(\%)$ \\
\hline - & - & - & - & - & - & - & - & - & - & -- \\
\hline & & E & 9,76 & 7,84 & 2,92 & 251 & 7,80 & $-3,24$ & 31,2 & 0,44 \\
\hline 6,3 & 3 & $\mathbf{R}$ & 9,63 & 7,55 & 3,00 & 243 & 7,57 & $-2,62$ & 28,9 & 0,31 \\
\hline & & E & 9,81 & 8,83 & 2,72 & 244 & 8,82 & $-3,27$ & 30,3 & 0,53 \\
\hline 7,6 & 3 & $\mathbf{R}$ & 9,66 & 8,47 & 2,78 & 239 & 8,50 & $-2,58$ & 28,2 & 0,31 \\
\hline & 0,6 & $\mathrm{E}$ & 9,72 & 18,81 & 1,80 & & & & & \\
\hline 15,2 & & $\mathrm{E}$ & 9,70 & 17,83 & 1,82 & 242 & 17,82 & $-3,11$ & 28,7 & 0,59 \\
\hline & 3 & $\mathbf{R}$ & 9,64 & 18,03 & 1,81 & 221 & 18,06 & $-2,61$ & 25,5 & 0,15 \\
\hline & & & & 31,76 & 1,42 & 195 & 31,71 & $-1,85$ & 19,7 & 0,14 \\
\hline 27,2 & 3 & E & & 31,9 & 1,40 & 210 & 31,77 & $-1,12$ & 20,0 & 0,28 \\
\hline & & & & 31,8 & 1,41 & 215 & 31,84 & $-1,73$ & 21,5 & 0,24 \\
\hline & 0,6 & E & 9,36 & 31,0 & 1,45 & & & & & \\
\hline 27,4 & & E & 9,37 & 29,89 & 1,45 & 205 & 29,85 & $-2,29$ & 21,7 & 0,24 \\
\hline & 3 & $\mathbf{R}$ & 9,22 & 30,62 & 1,44 & 201 & 30,62 & $-2,15$ & 20,9 & 0,13 \\
\hline & 0,6 & E & 8,77 & 41,90 & 1,29 & & & & & \\
\hline 35,5 & 2 & E & 8,79 & 41,69 & 1,30 & 167 & 41,71 & $-1,60$ & 15,4 & 0,20 \\
\hline & 3 & $\mathbf{R}$ & 8,55 & 41,55 & 1,29 & 165 & 41,52 & $-1,47$ & 14,7 & 0,11 \\
\hline 42,3 & 0,6 & $\mathbf{E}$ & 8,46 & 52,42 & 1,22 & & & & & \\
\hline 43,9 & 3 & $\mathbf{R}$ & 8,13 & 55,10 & 1,19 & 137 & 55,07 & $-0,74$ & 10,5 & 0,06 \\
\hline & & $\mathrm{E}$ & 7,91 & 58,56 & 1,18 & 163 & 58,51 & $-0,98$ & 12,3 & 0,09 \\
\hline 48 & 3 & $\mathbf{R}$ & 7,71 & 57,16 & 1,18 & 154 & 57,13 & $-1,15$ & 11,6 & 0,36 \\
\hline 52,5 & 0,6 & $\mathrm{E}$ & 7,54 & 65,95 & 1,14 & & & & & \\
\hline 55,6 & 3 & $\mathbf{R}$ & 7,40 & 71,9 & 1,12 & 112 & 71,92 & $-1,17$ & 7,1 & 0,55 \\
\hline 65,7 & 3 & $\mathbf{R}$ & 6,18 & 92,3 & 1,04 & & & & & \\
\hline
\end{tabular}

2.2 Dispositif DE MESURE. - Les mesures ont été effectuées au moyen d'un dispositif à température ajustable entre $4,2 \mathrm{~K}$ et l'ambiante que nous avons réalisé spécialement à cet effet. Il est constitué essentiellement d'une enceinte de mesure en cuivre, entourée d'isolant, et placée dans un dewar au-dessus d'un liquide cryogénique (Fig. 1). Le gaz froid provenant de ce bain ne peut s'échapper que par une rainure hélicoïdale pratiquée dans la paroi de l'enceinte. La température de celle-ci est stabilisée au moyen d'un régulateur S. E. I. N. piloté soit par une résistance de carbone de $390 \Omega$ Allen Bradley, soit par une résistance de platine de $400 \Omega$ à $0^{\circ} \mathrm{C}$, et agissant sur un enroulement chauffant en constantan. Afin d'augmenter la souplesse d'utilisation de l'appareil vers les hautes températures, la puissance maximale que peut fournir ce régulateur a été portée à $50 \mathrm{~W}$.

La stabilité et l'homogénéité de température à l'intérieur de l'enceinte ont été testées en mesurant la largeur de la transition résistive des échantillons de niobium et des fils d'alliages $\mathrm{Nb}-\mathrm{Hf}$. Ces transitions sont représentées sur la figure 2 ; on voit que certaines ont une largeur inférieure à $10^{-2} \mathrm{~K}$, ce qui indique qu'il n'y a vraisemblablement pas, dans l'enceinte, de gradient de température supérieur à cette valeur.

Le tube supportant l'enceinte de mesure, muni à sa partie supérieure d'une fermeture étanche, sert de passage à la canne porte-échantillons. A l'extrémité de celle-ci, les échantillons, qui pouvaient être au nombre de quatre à chaque expérience, étaient maintenus sur une plaque de cuivre, recouverte de mylar, par des pièces isolantes dans lesquelles venaient se visser les amenées de courant et prises de tension. Leur température était déterminée au moyen de résistances fixées également sur le porte-échantillons (Fig. 1). Celles-ci étaient, pour les températures inférieures à $20 \mathrm{~K}$, une résistance de carbone et au-dessus de $20 \mathrm{~K}$, une résistance de platine; nous avons estimé que la première donnait la température à $1 \%$ près et la seconde à $0,2 \mathrm{~K}$ près. Nous avons déterminé les températures critiques par la suite, avec une précision absolue de l'ordre de $0,02 \mathrm{~K}$, au moyen d'une résistance de germanium étalonnée au thermomètre à gaz.

Echantillons et thermomètres ont été mesurés par une méthode de quatre fils classique, en courant continu. Des alimentations électroniques stabilisées 


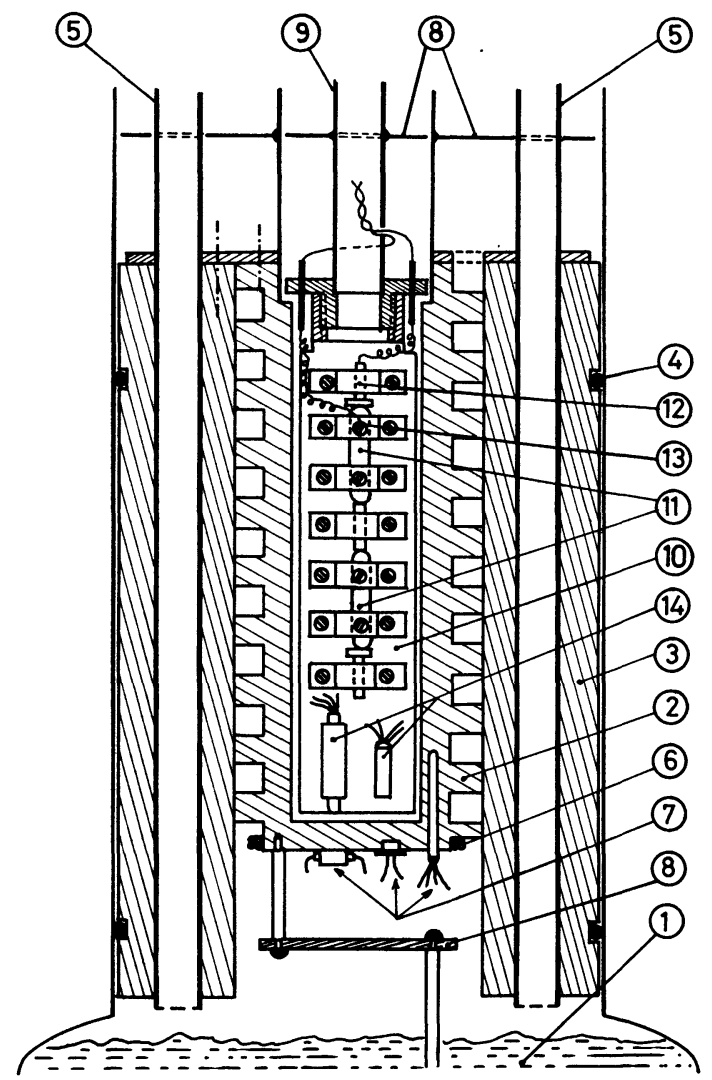

Fig. 1. - Schéma de la cellule de mesure. $1:$ hélium liquide, 2 : enceinte de mesure, $3:$ couronne en nylon, $4:$ joints toriques, $5:$ tube de remplissage en hélium et passage des connexions électriques, $6:$ enroulement chauffant, $7:$ thermomètres de régulation, 8 : écrans, 9 et $10:$ canne et plaque de cuivre porteéchantillons, 11 : échantillons, 12 : amenée de courant, $13:$ prise de tension, $14:$ thermomètres de mesure.

à mieux que $10^{-3}$ fournissaient les courants : $10 \mu \mathrm{A}$ dans la résistance de carbone et celle de germanium, $100 \mu \mathrm{A}$ dans la résistance de platine et $100 \mathrm{~mA}$ dans les échantillons (10 mA pour les mesures de $T_{\mathrm{c}}$ ). Pour mesurer les tensions, nous avons utilisé un potentiomètre Disselhorst Tinsley, la détection de zéro étant assurée par un amplificateur galvanométrique et un enregistreur Sefram. Nous avons tenu compte des f. é. m. parasites par une mesure en courant inverse ou à courant nul.

Pour les échantillons d'alliages $\mathrm{Nb}-\mathrm{Hf}$, nous estimons que l'incertitude sur les valeurs de résistivité est au maximum 3,5\%. La plus grande partie de celle-là provient de la mesure des dimensions : $1 \%$ sur la section et $2 \%$ sur la longueur. Mais cette dernière erreur se trouve pratiquement éliminée lorsque l'on compare les échantillons entre eux : en effet, les prises de tension étaient fixes et distantes de $10 \mathrm{~mm}$ pour tous les échantillons de diamètre $3 \mathrm{~mm}$. Pour permettre également de les comparer avec le niobium, l'échantillon $\mathrm{Nb} 1$ a été mesuré dans les mêmes conditions ; de plus, nous avons normalisé sa résistivité à la valeur donnée par White et Woods [13] : $\rho(295)-\rho_{0}=14,5 \mu \Omega$.cm, et répercuté cette opération sur tous les échantillons précédents.
3. Résultats et discussion préliminaire. -3.1 NiOBIUM. - La résistivité des échantillons $\mathrm{Nb} 1$ et $\mathrm{Nb} 2$, que nous avons mesurée entre l'ambiante et leur température critique, était à $9,5 \mathrm{~K}$ (c'est-à-dire juste audessus de $T_{\mathrm{c}}$ ) proche de sa valeur résiduelle. Nous avons alors déterminé cette dernière par extrapolation. Le rapport $\rho(300) / \rho(9,5)$ valait 97 pour $\mathrm{Nb} 1$ et 223 pour $\mathrm{Nb} 2$, ce qui donnait $\rho(300) / \rho_{0}$ égal à 99 et 238 , respectivement.

Nous avons obtenu une température critique de $9,29 \mathrm{~K}$ pour l'échantillon $\mathrm{Nb} 1$ et $9,27 \mathrm{~K}$ pour $\mathrm{Nb} 2$, en accord avec les valeurs données pour des échantillons de rapport voisin $[14,15]$. Cependant, ces transitions supraconductrices présentaient, comme on le voit sur la figure 2, une largeur de plusieurs centièmes de degrés. Cela résultait certainement de la diffusion de gaz dans les échantillons qui avaient séjourné à l'air avant la mesure. Nous avons vérifié ce fait sur l'échantillon $\mathrm{Nb3}$, dont le rapport $\rho(300) / \rho_{0}$ était égal à 167 . Immédiatement après recuit, sa largeur de transition était de quelques $\mathrm{mK}$; elle est devenue de l'ordre de grandeur des précédentes après que l'échantillon ait séjourné quelques semaines à l'air (cf. Fig. 2).

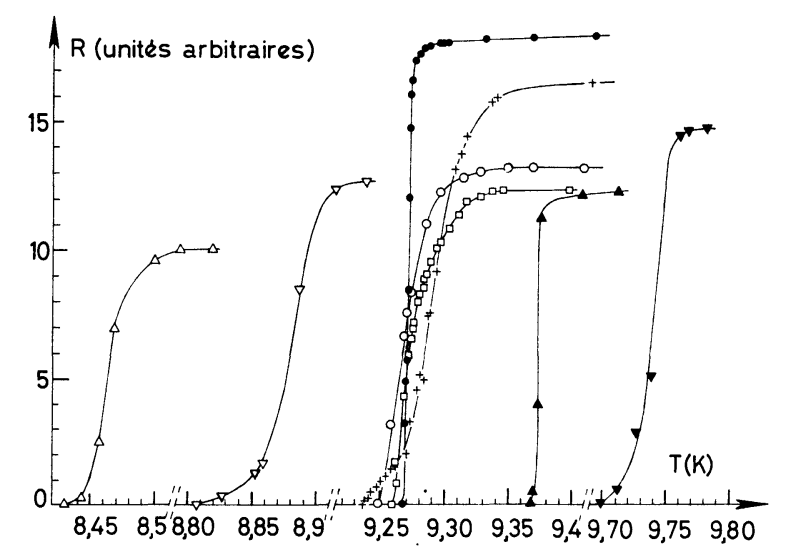

Fig. 2. - Largeurs de transition supraconductrices. + Nb1, $\bigcirc \mathrm{Nb} 2$, $\mathrm{Nb} 3$ immédiatement après recuit, $\square \mathrm{Nb} 3$ après vieillissement à l'air. $\mathbf{\nabla}, \mathbf{\Delta}, \nabla, \Delta$ fils $\mathrm{Nb}-\mathrm{Hf}$ écrouis de concentration respective $15,2 \%, 27,4 \%, 35,5 \%$ et $42,3 \%$ at. $\mathrm{Hf}$.

La littérature fournit des valeurs très diverses de la résistivité du niobium à l'ambiante. Celle qui demeure la plus connue, bien qu'elle ait été déterminée sur des échantillons dont le meilleur avait un rapport $\rho(295) / \rho_{0}$ de 31,5 , est la valeur de White et Woods [13] que nous avons indiquée ci-contre. Dans tout ce qui suit, nous désignerons par $\rho_{\mathbf{i}}(T)$ la quantité $\rho(T)-\rho_{0}$. White et Woods obtenaient donc $\rho_{\mathrm{i}}(295)=14,5 \mu \Omega$.cm, mais la mesure d'échantillons plus purs a donné généralement des valeurs inférieures à celle-ci. Ainsi, on peut relever dans les résultats de Clinard et Kempter [16] : $\rho(295) \simeq 14,5 \mu \Omega$.cm pour $\rho(295) / \rho_{0} \simeq 50$, ce qui conduit à $\rho_{\mathbf{i}}(295) \simeq 14,2 \mu \Omega . \mathrm{cm}$, tandis que du tableau $\rho(T)$ fourni par Abraham et Deviot [17] on tire, par interpolation $\rho(295)=14,34 \mu \Omega$.cm, pour un 
échantillon de rapport $\rho(295) / \rho(20)$ relativement élevé (dans ce cas $\rho$ n'a pas été mesuré au-dessous de $20 \mathrm{~K}$ ).

Nos résultats bruts donnaient $\rho_{\mathrm{i}}(295)$ égal à $14,6 \mu \Omega$.cm pour $\mathrm{Nb} 1$ et 14,1 pour $\mathrm{Nb} 2$, ce qui, compte tenu de notre marge d'erreur, était compatible avec les valeurs précédentes. Nous avons alors choisi de normaliser la résistivité de l'échantillon' $\mathrm{Nb} 2$, comme celle de $\mathrm{Nb} 1$ à la valeur $\rho_{\mathrm{i}}(295)=14,5 \mu \Omega . \mathrm{cm}$. Webb [8], dont les échantillons avaient un rapport $\rho(300) / \rho_{0}$ allant de 11000 à 16500 , a signalé également qu'une mauvaise définition de leurs dimensions géométriques ne lui permettait pas de calculer une valeur précise de leur résistivité. Nous avons donc appliqué la même normalisation à tous ses résultats numériques que nous citerons par comparaison.

Nous avons représenté en coordonnées logarithmiques sur la figure 3 les valeurs de $\rho_{\mathrm{i}}(T)=\rho(T)-\rho_{0}$ que nous avons obtenues ainsi que celles de White et Woods et de Webb. Bien que ces courbes soient la somme de plusieurs termes variant différemment avec $T$ (Webb a montré qu'à basse température ses résultats étaient de la forme

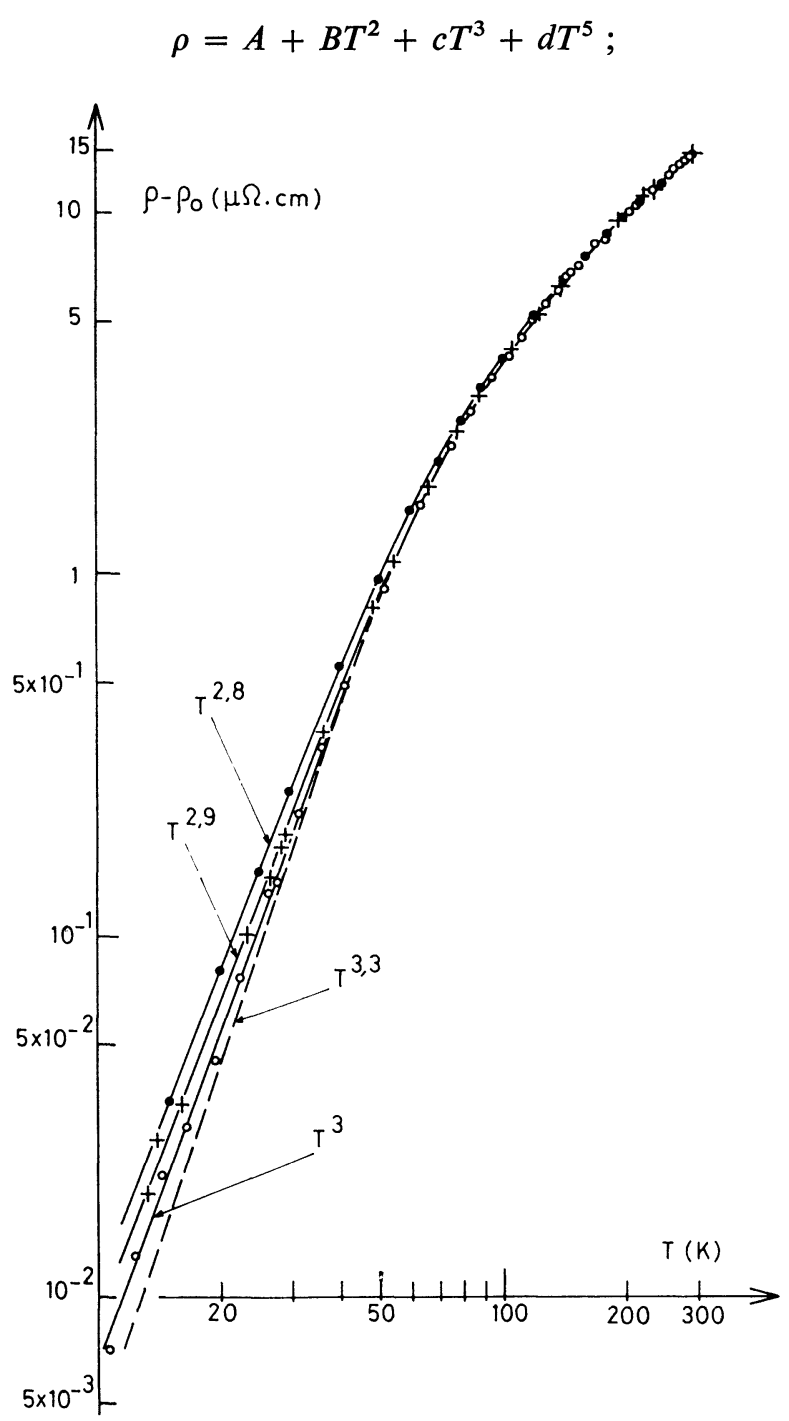

FIG. 3. - Variation de $\rho(T)-\rho_{0}$ du niobium. + Nb1, $\bigcirc \mathrm{Nb} 2$, White et Woods [13], - - Webb [8]. nous reviendrons sur ce point au chapitre suivant), on peut selon le procédé classique, approximer leur partie basse température par une courbe en $T^{n}$. D'après la figure 3 , on peut ainsi, pour $T<40 \mathrm{~K}$, approximer les résultats de White et Woods par l'expression $\rho_{\mathrm{i}} \simeq 1,8 \times 10^{-5} \cdot T^{2,8}$, la résistivité de l'échantillon $\mathrm{Nb} 1$ par $1,1 \times 10^{-5} . T^{2,9}$, celle de l'échantillon $\mathrm{Nb} 2$ par $6,8 \times 10^{-6} \cdot T^{3}$ et la courbe de Webb par $2,2 \times 10^{-6} \cdot T^{3,3}$ (en fait White et Woods ont indiqué $n \simeq 2,7$ et Webb $n \simeq 3,2$ ). Par ailleurs, Clinard et Kempter [16] donnent la courbe $\rho_{\mathrm{i}}=9,4 \times 10^{-6} \cdot T^{2,94}$ pour $T<47 \mathrm{~K}$, non représentée sur la figure 3 car très proche de celle de l'échantillon Nb1. La figure 3 montre que la valeur de $\rho_{\mathrm{i}}$ la plus faible à basse température est celle des échantillons de Webb, qui sont ceux dont le rapport de résistivité est le plus élevé. Il est vraisemblable que les différences entre celle-ci et les autres courbes de la figure 3 constituent des écarts à la règle de Matthiessen. Ceux-ci seront calculés et discutés au chapitre suivant.

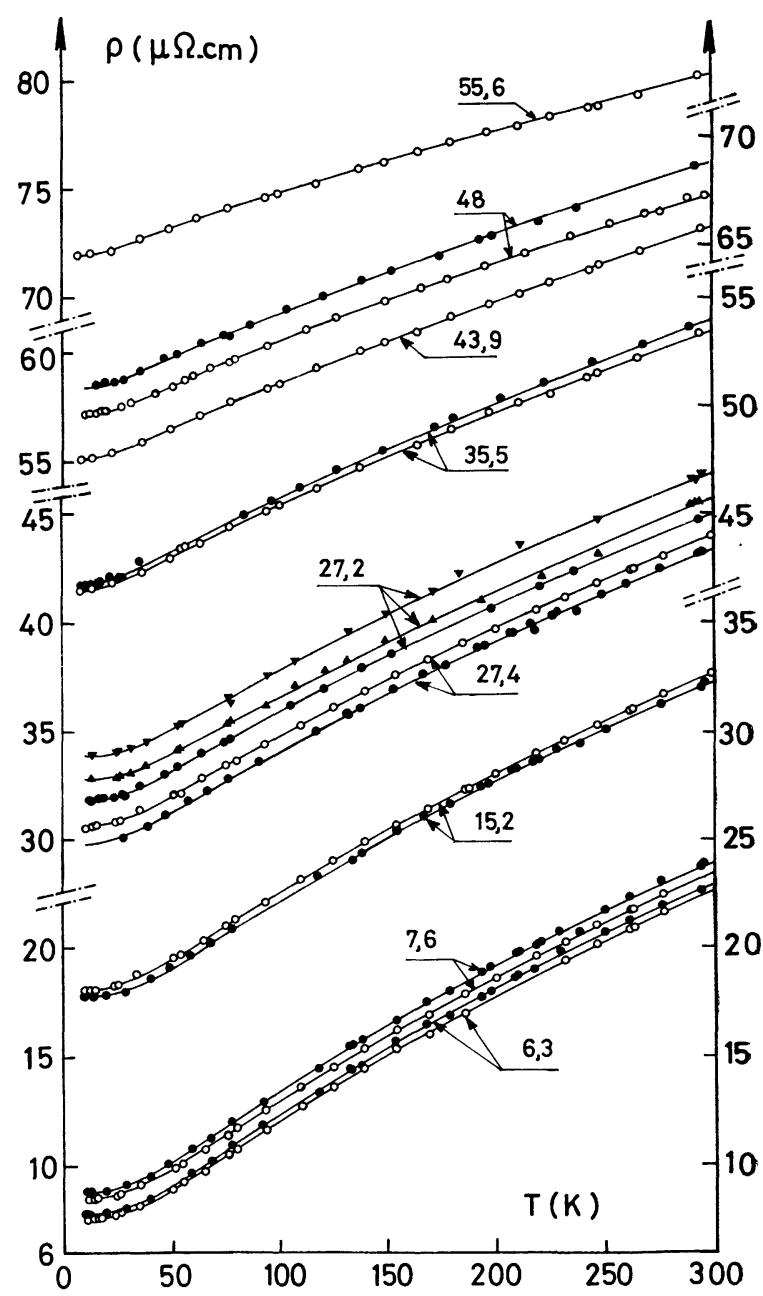

Fig. 4. - Résistivité des alliages $\mathrm{Nb}-\mathrm{Hf}$ en fonction de la température : $\bigcirc$ échantillons recuits; $\boldsymbol{\bullet}, \boldsymbol{\nabla}, \boldsymbol{\Delta}$ échantillons écrouis ; $\Delta, \boldsymbol{\nabla}$ courbes décalées vers le haut respectivement de $1 \mu \Omega$.cm et $2 \mu \Omega$.cm. Les courbes sont celles calculées par la relation (9). Les chiffres indiquent la concentration en \% atomiques de Hf. 
3.2 Alliages niobium-hafnium. - Les résultats expérimentaux de résistivité sont représentés sur la figure 4. Pour quelques échantillons, nous avons tracé $\rho(T)-\rho_{0}$, sur la figure 5 . On voit que ces quantités présentent des écarts importants à température ambiante. Mais on peut dire qu'à basse température elles se confondent, dans la limite de précision expérimentale, en une courbe moyenne, d'équation

$$
\rho_{\mathrm{i}} \simeq 5 \times 10^{-5} \cdot T^{2,7} \text {. }
$$

Les écarts de ces courbes par rapport au niobium seront commentés au chapitre 4 .

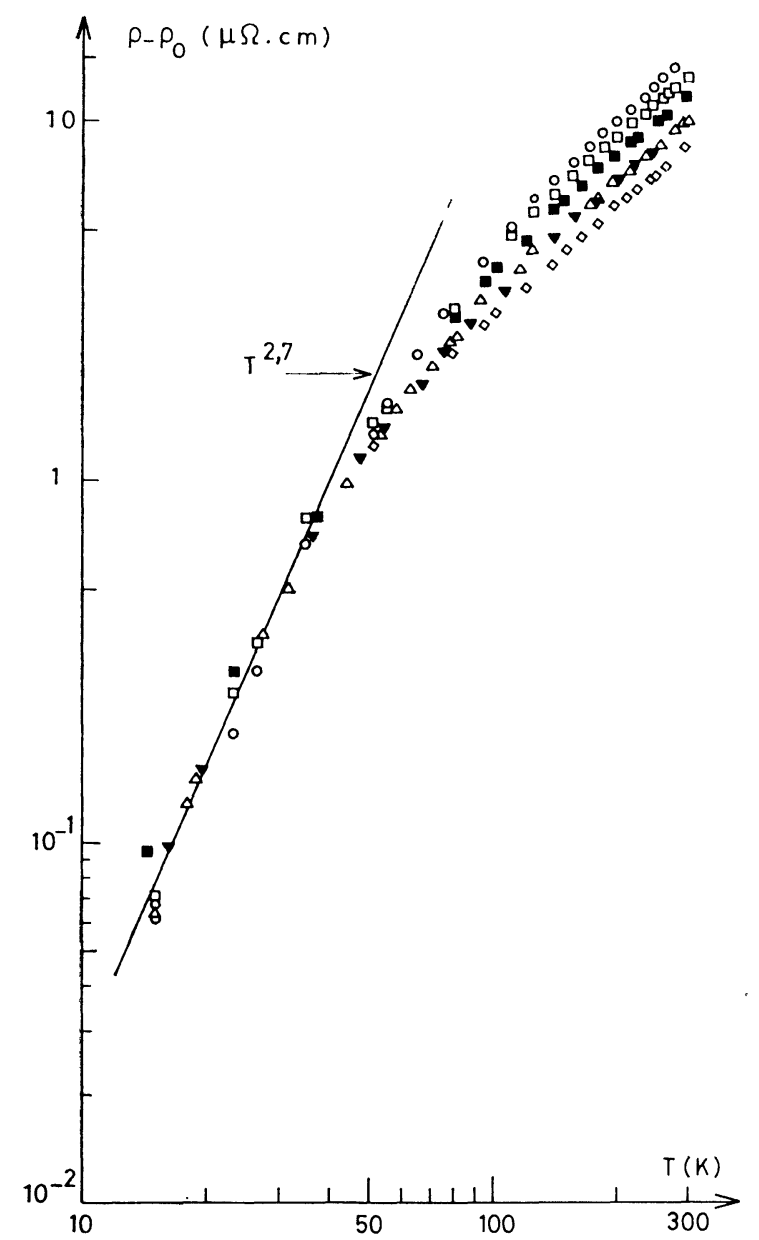

Fig. 5. - Variation de $\rho(T)-\rho_{0}$ de quelques alliages $\mathrm{Nb}-\mathrm{Hf}$. $\bigcirc 6,3 \%$ et $7,6 \%, \square 27,4 \%, \square 35,5 \%, \triangle 48 \%$ recuits, $\nabla 48 \%$ écroui, $\diamond 55,6 \%$ recuit.

Les températures critiques, résistivités résiduelles et rapports de résistivité que nous avons obtenus pour les alliages $\mathrm{Nb}-\mathrm{Hf}$ sont donnés dans le tableau I.

Nos résultats de résistivité résiduelle sont représentés sur la figure 6, en compagnie de ceux de Berlincourt et Hake [3], et de Siemens et al. [4]. Ces auteurs, qui ont mesuré des fils écrouis, ont obtenu des valeurs supérieures aux nôtres. Ces écarts ne peuvent être expliqués par des variations du taux d'écrouissage, puisque dans nos échantillons celles-ci ont un effet assez faible. Ils peuvent encore être dûs aux incertitudes expérimen-

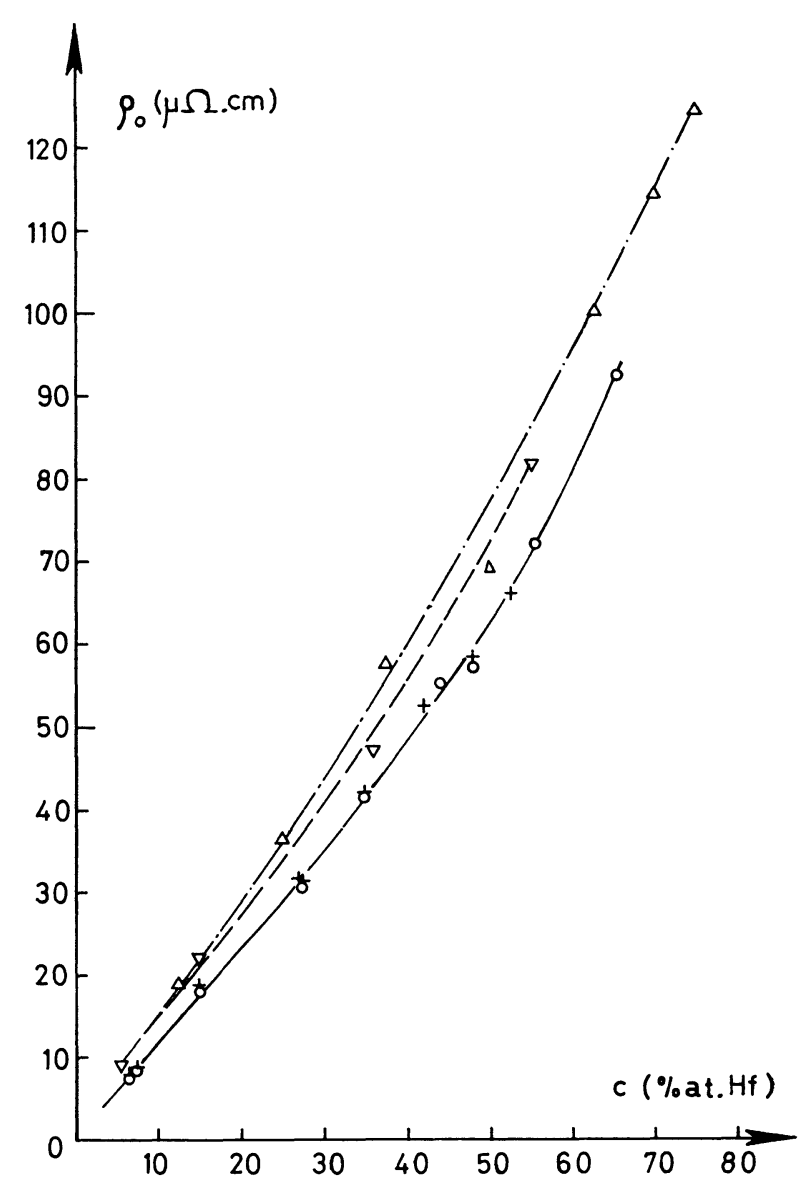

Fig. 6. - Résistivité résiduelle des alliages Nb-Hf. Trait plein : nos échantillons ( + écrouis, $\bigcirc$ recuits). $-. \triangle-.-$ Berlincourt et Hake [3]. $---\nabla--$ Siemens et al. [14].

tales, qui étaient particulièrement élevées dans la méthode utilisée par Berlincourt et Hake, aux différences de pureté des échantillons, ou peut-être, pour les concentrations élevées, à la présence d'une quantité plus ou moins faible de précipités. Dans les alliages $\mathrm{Nb}$-Hf, comme dans la plupart des alliages du même type, $\rho_{0}$ augmente rapidement avec la concentration ; ce fait a été observé par Berlincourt et Hake [3] puis par d'autres auteurs, sur $\mathrm{Nb}-\mathrm{Ti}$ [18], $\mathrm{Nb}-\mathrm{Zr}$ [19], Ta-Ti [20]. En même temps on constate que le rapport $\rho(300) / \rho_{0}$ décroît rapidement avec la concentration. Ce dernier peut même devenir inférieur à l'unité dans $\mathrm{Nb}-\mathrm{Ti}$ [18], Nb-Zr [21], V-Ti [22], Mo-Ti [1], mais alors cela est dû à la précipitation de phase $\omega$ [1]. Ce phénomène n'a pas été observé jusqu'à présent dans les alliages de hafnium, mais on doit se demander s'il a pu se produire dans les alliages à 70 et $75 \%$ de Berlincourt et Hake, dont la résistivité résiduelle était particulièrement élevée. Nous avons mesuré le rapport d'un échantillon à $65,7 \%$ at. Il était seulement de 1,04. Mais il nous était impossible (étant donné leur mode de préparation : trempe à partir de $1000^{\circ} \mathrm{C}$ seulement) d'obtenir des alliages plus concentrés exempts de précipités de phase $\alpha$ et, par suite d'apporter des éclaircissements sur ce point. 
Nous avons tracé sur la figure 7 la température critique de nos échantillons en fonction de la concentration, conjointement avec les résultats de Hulm et Blaugher [2] obtenus sur des échantillons recuits ou bruts de fusion, ceux de Siemens et al. [4], et la valeur donnée par Koch et Carpenter [7] pour un alliage à $38 \%$ at. trempé à partir d'une température de recuit de $1800^{\circ} \mathrm{C}$. Cette dernière valeur, ainsi que celles de l'échantillon à $50 \%$ de Hulm et Blaugher et de notre échantillon à $55,6 \%$ mises à part, il y a, dans le cas des

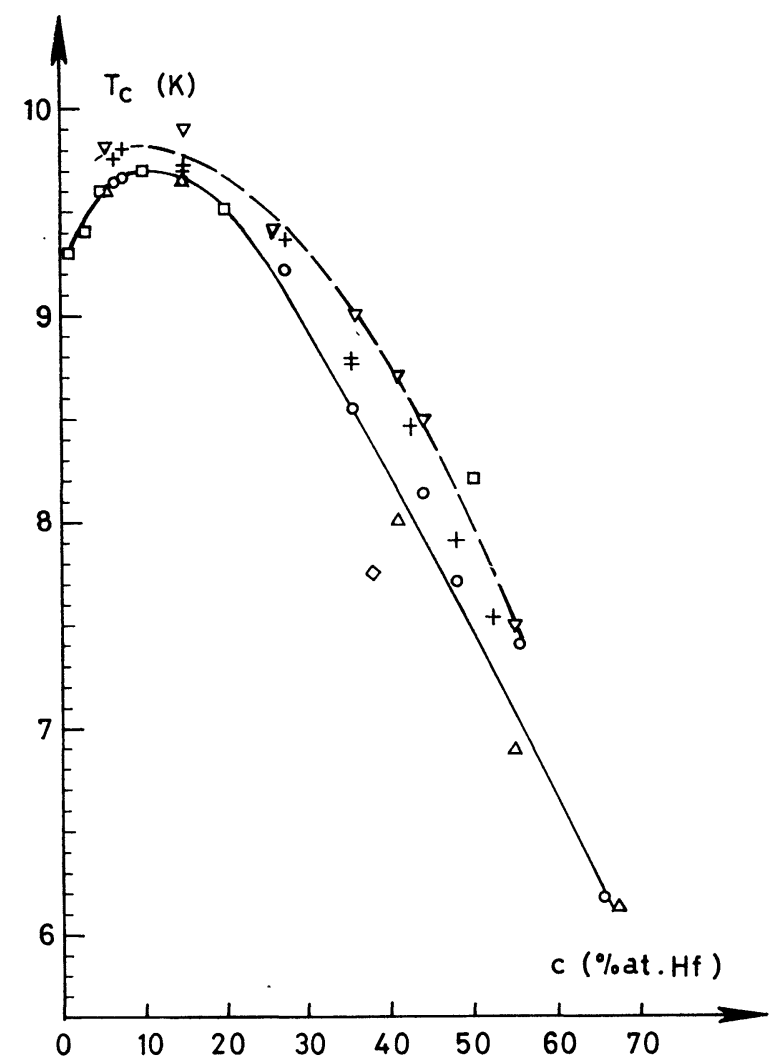

FIG. 7. - Température critique des alliages Nb-Hf. $+, \mathrm{O}:$ nos échantillons ( + écrouis, $\bigcirc$ recuits). $\square$ Hulm et Blaugher [2]. $\nabla, \triangle$ Siemens et al. $[4]$ ( $\nabla$ écrouis, $\triangle$ recuits). $\diamond$ Koch et Carpenter [7]. Les courbes en trait plein et discontinu représentent une valeur moyenne, respectivement pour les échantillons recuits et écrouis.

échantillons recuits, un bon accord entre les différentes mesures. Leur température critique passe par un maximum au voisinage de $10 \%$ at. On observe également un tel maximum, mais chaque fois pour une concentration différente, dans les systèmes d'alliages voisins; ce point a été commenté par Hulm et Blaugher [2]. La température critique des échantillons écrouis est toujours supérieure à celle des échantillons recuits. Cette augmentation peut atteindre $10 \%$, mais on ne peut pas la relier uniquement au taux d'écrouissage. En effet d'autres facteurs, liés ou non au précédent, ont pu intervenir dans les valeurs de $T_{\mathrm{c}}$ observées : inhomogénéités de concentration, ou peut-être, pour les concentrations élevées, précipitation de phase $\alpha$. Notons que ce dernier phénomène, en appauvrissant en hafnium la matrice, doit provoquer une élévation du $T_{\mathrm{c}}$ de celle-ci, et aussi un abaissement de sa résistivité ; mais une telle corrélation ne semble pas exister dans nos échantillons, même à forte concentration.

4. Analyse mathématique des résultats de résistivité et discussion. - 4.1 LOI DE VARIATION AVEC LA TEMPÉRATURE. - Au chapitre précédent, nous avons, selon l'interprétation classique, séparé la résistivité en deux termes : la résiduelle, $\rho_{0}$, considérée comme indépendante de $T$, et la contribution dépendant de la température, $\rho_{\mathrm{i}}(T)$. Mais cette dernière peut ellemême être la somme de plusieurs contributions dont la séparation nécessite une analyse plus détaillée des résultats; les différents mécanismes de diffusion des électrons à envisager ici sont ceux qui apparaissent ordinairement dans les métaux de transition non magnétiques et dont la description est classique [23, 24]. Il s'agit tout d'abord de l'interaction des électrons $\mathrm{s}$ avec les phonons, donnée par la relation de BlochGrüneisen, qui peut s'écrire :

$$
\rho_{\mathrm{i}, \mathrm{s}-\mathrm{s}}(T) \propto\left(\frac{T}{\theta}\right)^{5} J_{5}\left(\frac{\theta}{T}\right)
$$

où $\theta$ est une température de Debye et $J_{5}(\theta / T)$ une intégrale de la famille

$$
J_{n}(x)=\int_{0}^{x} z^{n} e^{z}\left(e^{z}-1\right)^{-2} \mathrm{~d} z .
$$

D'après cette relation, $\rho_{\mathrm{i}, \mathrm{s}-\mathrm{s}}(T)$ doit être proportionnelle à $T^{5}$ à basse température, et à $T$ à haute température. C'est ensuite la contribution due aux transitions s-d que Wilson [25] a proposé d'exprimer sous la forme :

$$
\rho_{\mathrm{i}, \mathrm{s}-\mathrm{d}}(T) \propto \int_{\theta_{\min / T}}^{\theta / T} z^{3} e^{z}\left(e^{z}-1\right)^{-2} \mathrm{~d} z
$$

où $\theta_{\min }$ est défini par le vecteur d'onde de phonon minimum nécessaire pour donner lieu à une transition s-d. Cette relation prévoit que $\rho_{\mathrm{i}, \mathrm{s}-\mathrm{d}}(T)$ doit, à haute température, être linéaire en $T$ comme $\rho_{\mathrm{i}, \mathrm{s}-\mathrm{s}}$ et, à basse température, varier en $T^{3}$ pour $T>\theta_{\min }$ et exponentiellement pour $T<\theta_{\min }$. Enfin, il peut encore exister dans la résistivité des métaux de transition, une contribution d'interaction électron-électron en $T^{2}$, visible à basse température, et des effets de non-linéarité à haute température. La relation (1), à elle seule, a permis d'analyser avec succès la résistivité de nombreux métaux, mesurée entre la température de l'hélium liquide et l'ambiante. Meaden [24] a répertorié différentes méthodes utilisées pour cela et cité les températures de Debye obtenues; celles-ci sont en plus ou moins bon accord avec celles déduites de la chaleur spécifique. On a même appliqué cette relation à la résistivité de métaux de transition, mais avec de médiocres résultats.

Dans le cas particulier du niobium, plusieurs auteurs ont analysé sa résistivité à basse température. Evans et Erickson [19] ont simplement approximé celle-ci ainsi 
que celle d'alliages $\mathrm{Nb}-\mathrm{Zr}$, entre $35 \mathrm{~K}$ et l'ambiante, par une droite, dont ils ont écrit l'équation sous la forme $R(T) / R(\theta)=a T / \theta+b$. Cela est conforme à l'expression (1) d'après laquelle $\rho_{\mathrm{i}}(T) / \rho_{\mathrm{i}}(\theta)$ doit être proportionnel à $T / \theta$ dans la région de température où $\rho_{\mathrm{i}}(T)$ est proche de la linéarité. Ils ont déterminé les paramètres $a$ et $b$ en utilisant des valeurs de $\theta$ données par la chaleur spécifique à basse température, mais ils n'ont pu tirer aucune conclusion de la variation de ces paramètres avec la concentration, car les approximations faites étaient trop grossières. Clinard et Kempter [16], qui ont mesuré la résistivité du niobium et autres métaux de transition, ainsi que de leurs carbures, au-dessous de l'ambiante, approximaient la partie basse température des résistivités idéales par une courbe en $T^{n}$ et appliquaient à la partie haute température une expression analogue à 1 . Dans le cas du niobium, ils obtenaient, pour $T<47 \mathrm{~K}$, l'expression déjà citée : $\rho_{\mathrm{i}}=9,4 \times 10^{-6} \cdot T^{2,94} \mu \Omega$.cm et pour $T>47 \mathrm{~K}: \rho_{\mathrm{i}}=45(T / \theta)^{5} J_{5}(\theta / T)$, avec $\theta=223 \mathrm{~K}$. Ils séparaient ainsi arbitrairement le domaine de température $T_{\mathrm{c}}$ - ambiante en deux intervalles, ce qui au point de vue mathématique permet de décrire les résultats expérimentaux de façon relativement satisfaisante, mais ne paraît pas justifié physiquement.

Finalement, Webb [8] a montré que la résistivité du niobium peut être représentée de $2 \mathrm{~K}$ à l'ambiante, par une expression de la forme :

$$
\begin{aligned}
\rho=A+B T^{2}+C\left(\frac{T}{\theta}\right)^{3} J_{3}\left(\frac{\theta}{T}\right) & + \\
& +D\left(\frac{T}{\theta}\right)^{5} J_{5}\left(\frac{\theta}{T}\right) .
\end{aligned}
$$

Dans cette relation, la constante $A$ doit être égale à la résistivité résiduelle ; $B T^{2}$ est une contribution due à l'interaction électron-électron. On retrouve les deux termes d'interaction électron-phonon évoqués plus haut; celui en $T^{5}$ est identique à l'expression (1) tandis que celui en $T^{3}$ est l'expression (2), simplifiée en faisant $\theta_{\min }=0$. Webb a longuement discuté ce terme en $T^{3}$, car il peut paraître trop schématique pour représenter convenablement $\rho_{\mathrm{s}-\mathrm{d}}$. En effet, l'expression (2) a été établie en supposant en particulier deux surfaces de Fermi $\mathrm{s}$ et $\mathrm{d}$ sphériques et sans intersection, et Ziman [23] précise que dans d'autres cas on devrait trouver des expressions de $\rho_{\mathrm{s}-\mathrm{d}}$ variant en $T^{4}$ ou $T^{5}$ à basse température. Or, la surface de Fermi du niobium, telle qu'elle a été calculée par Mattheis [26], est loin de posséder la forme simple requise par l'expression (2). Mais Webb justifie l'emploi du terme en $T^{3}$ en évaluant $\theta_{\min }$ à environ $10 \mathrm{~K}$; ce terme se justifie surtout par le fait que la variation de $\rho_{\mathrm{i}}(T)$ qu'il a observée est pratiquement en $T^{3}$ à basse température et que l'ensemble de l'expression (3) représente correctement ses résultats expérimentaux.

Cependant, avec les valeurs numériques des paramètres $A, B, C, D, \theta$ donnés par Webb, l'expression (3) ne serait pas suffisante pour représenter la résistivité du niobium au-dessus de $300 \mathrm{~K}$, car le terme $B T^{2}$, que Webb trouve faible, mais positif, donnerait une courbure positive à $\rho(T)$. Or, les mesures de Abraham et Deviot [17] et de Cesairliyan [27] montrent que la résistivité du niobium au-dessus de $300 \mathrm{~K}$ présente au contraire une concavité tournée vers le bas. Cet effet apparaît déjà nettement au voisinage de l'ambiante ; il est encore plus accentué dans la résistivité des alliages $\mathrm{Nb}-\mathrm{Hf}$. C'est un comportement que l'on rencontre dans la résistivité des métaux de transition et qui a été interprété par Mott, en tenant compte de la variation de la densité d'états $N_{\mathrm{d}}$ avec l'énergie $E$, au voisinage du niveau de Fermi $E_{\mathrm{F}}$ (cf. Mott et Jones [28]). Le résultat de cette variation est de multiplier la résistivité par un facteur que, avec Meaden [24], on peut écrire

$$
\begin{array}{r}
1-\frac{\pi^{2}}{6}(k T)^{2}\left[3\left(\frac{1}{N_{\mathrm{d}}} \frac{\partial N_{\mathrm{d}}}{\partial E}\right)^{2}-\frac{1}{N_{\mathrm{d}}} \frac{\partial^{2} N_{\mathrm{d}}}{\partial E^{2}}\right]_{E=E_{\mathrm{F}}} \equiv \\
\equiv 1+a T^{2}
\end{array}
$$

où $k$ est la constante de Boltzmann. Cet effet, s'il n'est visible en général qu'à des températures supérieures à l'ambiante, a lieu à toute température et l'on peut dire que la résistivité de type s-d devient de la forme

$$
\left[A+C\left(\frac{T}{\theta}\right)^{3} J_{3}\left(\frac{\theta}{T}\right)\right]\left(1+a T^{2}\right),
$$

si bien qu'au lieu de l'expression (3), on obtient

$$
\begin{aligned}
\rho=A+B T^{2}+C\left(\frac{T}{\theta}\right)^{3} J_{3}\left(\frac{\theta}{T}\right) & +D\left(\frac{T}{\theta}\right)^{5} J_{5}\left(\frac{\theta}{T}\right)+ \\
& +E T^{2}\left(\frac{T}{\theta}\right)^{3} J_{3}\left(\frac{\theta}{T}\right)
\end{aligned}
$$

Dans cette nouvelle expression, l'effet considéré est représenté à la fois par le dernier terme et par une contribution $a A T^{2}$ qui se trouve incluse dans le terme $B T^{2}$. Celui-ci conserve en même temps sa signification première qui est de représenter la contribution positive, due à l'interaction électron-électron. Dans le cas du niobium et des alliages $\mathrm{Nb}-\mathrm{Hf}$, le coefficient $a$ doit être négatif et par suite $E$ également ; le paramètre $B$ sera lui aussi négatif si l'effet en question l'emporte sur la contribution électron-électron. Mais, s'agissant d'un effet du second ordre, il ne paraît pas nécessaire de le représenter par deux termes dont la valeur sera forcément faible. Nous allons voir précisément qu'il sera suffisant de conserver l'un ou l'autre des termes négatifs $B T^{2}$ et $E T^{2}(T / \theta)^{3} J_{3}(\theta / T)$ pour représenter correctement nos résultats. Le premier sera en général le plus adéquat ; cela se justifie, non seulement parce que ce terme est le plus simple, mais, comme il provient du produit $a A T^{2}$, il doit être plus important que le second dans les alliages, où la valeur de $A$ est élevée.

4.2 RÉSultats ET Discussion. - La méthode de calcul adoptée consistait, partant des couples de valeurs 
$(\rho, T)$ expérimentales, à calculer par moindres carrés linéaires les coefficients $A, B, C, D$ et $E$ pour différentes valeurs de $\theta$ successives. La valeur de $\theta$ choisie était celle qui correspondait au minimum de l'écart quadratique moyen relatif, que nous avons défini par

$$
\sigma=\sqrt{\frac{1}{n} \sum_{q=1}^{n}\left(\frac{\rho_{q \mathrm{c}}-\rho_{q \mathrm{~m}}}{\rho_{q \mathrm{~m}}}\right)^{2}}
$$

où $n$ est le nombre total de points et $\rho_{q \mathrm{c}}$ et $\rho_{q \mathrm{~m}}$ sont les valeurs de $\rho$ au point $q$, respectivement calculée et mesurée. Dans le cas du niobium et des alliages à 6,3\%, $7,6 \%$ et $15,2 \%$, chaque valeur de $\rho$ était affectée d'un poids qui lui était inversement proportionnel.

Pour le niobium comme pour les alliages $\mathrm{Nb}-\mathrm{Hf}$, nous avons tout d'abord essayé la relation (5) complète mais constaté que le nombre de paramètres pouvait être réduit, parce que l'un d'eux se révélait nul ou prenait une valeur aberrante (valeur négative pour $C$ ou $D$ par exemple). Nous avons alors essayé systématiquement des formes simplifiées de la relation (5) en supprimant un ou plusieurs de ses termes. La forme finalement retenue était celle qui donnait le minimum minimorum de $\sigma$.

4.2.1 Niobium. - Pour nos deux échantillons de niobium, c'est une relation qui ne comporte pas de terme en $T^{2}$ :

$$
\begin{aligned}
\rho=A+C\left(\frac{T}{\theta}\right)^{3} J_{3}\left(\frac{\theta}{T}\right)+ & D\left(\frac{T}{\theta}\right)^{5} J_{5}\left(\frac{\theta}{T}\right)+ \\
& +E T^{2}\left(\frac{T}{\theta}\right)^{3} J_{3}\left(\frac{\theta}{T}\right)
\end{aligned}
$$

avec $E$ négatif, qui a donné la plus faible valeur de $\sigma$. L'expression (3), avec cette fois $B$ négatif, donne des résultats presque aussi bons. Nous avons réuni dans le tableau II la valeur des différents paramètres que nous avons obtenus avec chacune de ces deux expressions, les valeurs correspondantes de $\sigma$ et de l'écart maximal (qui a lieu à basse température) ainsi que les résultats de Webb.

A basse température, les expressions (3) et (6) se réduisent respectivement à

$$
\rho=A+B T^{2}+c T^{3}+d T^{5}
$$

et

$$
\rho=A+c T^{3}+(d+e) T^{5}
$$

et nous donnons dans le tableau II les valeurs des paramètres $c, d$ et $e$ déduites de celles de $C, D$ et $E$. Cette simplification résulte du fait que $J_{3}(\theta / T)$ et $J_{5}(\theta / T)$ tendent chacune vers une limite (à savoir : 7,212 et 124,4 , respectivement). Mais si l'on veut que ces intégrales diffèrent de moins de $1 \%$ de cette limite, on doit avoir $\theta / T \gtrsim 10$ pour la première et $\theta / T \gtrsim 13$ pour la seconde; avec les valeurs de $\theta$ qui figurent dans le tableau II les relations (7) et (8) ne sont donc valables qu'au-dessous de $20 \mathrm{~K}$ environ. Notons qu'elles donnent alors une variation de $\left(\rho_{\text {calculé }}-A\right)$ plus rapide que $T^{3}$, tandis que pour nos échantillons, nous avions assimilé $\rho_{\mathrm{i}}$ à des courbes en $T^{2,9}$ et $T^{3}$. Mais nous avions pour cela pris en compte surtout des points à $T>20 \mathrm{~K}$, où la variation de $\rho_{\mathrm{i}}$ est moins rapide que celle indiquée par les expressions (7) et (8). De plus, $A$ n'est pas rigoureusement égal à $\rho_{0}$, et l'on retrouve pratiquement le tracé de $\rho(T)-\rho_{0}$ fait sur la figure 3, si l'on considère $\left(\rho_{\text {calculé }}-\rho_{0}\right)$ et non $\left(\rho_{\text {calculé }}-A\right)$. On voit ainsi l'ampleur de l'approximation faite précédemment en assimilant, jusqu'à $40 \mathrm{~K}$ ou plus, $\rho(T)-\rho_{0}$ à une simple courbe en $T^{n}$.

Les valeurs de $\theta$ déterminées au moyen de l'expression (6), compte tenu de leur précision, que nous estimons à $\pm 15 \mathrm{~K}$, environ, sont en bon accord avec celles obtenues par mesure de chaleur spécifique à très basse température : pour $T<3 \mathrm{~K}$, ces dernières vont de $275 \mathrm{~K}$ à $280 \mathrm{~K}[14,15,29,30]$; il y a également accord avec celle déterminée par Linkoaho [31] : $284 \pm 6 \mathrm{~K}$, au moyen de la réflexion de rayons $X$. La température de Debye calculée par Webb, pour laquelle il annonce une précision de $\pm 10 \mathrm{~K}$, était elle-même en très bon accord avec les précédentes.

Nous donnons dans le tableau II la proportion à $300 \mathrm{~K}$, par rapport à la résistivité totale, des différents termes dépendant de $T$. On voit que dans le cas de Webb, celle de $B T^{2}$ est très faible ; en fait, la valeur de $B$ obtenue par Webb, en appliquant la relation (3) se situait, pour une variation de $\theta$ de $\pm 10 \mathrm{~K}$, dans une fourchette de $\pm 4,4 \times 10^{-6}$, soit, en valeur absolue, plus de 10 fois la valeur de $B$ elle-même. En appliquant l'expression (7) (dans laquelle il négligeait le terme $\mathrm{d} T^{5}$ ) à ses résultats pour $T \lesssim 9 \mathrm{~K}$, Webb a par contre observé un terme $B T^{2}$ positif ; avec la valeur maximale $B=8,2 \times 10^{-6}$ qu'il a ainsi déterminée, le terme $B T^{2}$ représente $15 \%$ de la résistivité totale à $10 \mathrm{~K}, 7,3 \%$ à $20 \mathrm{~K}$ et $5 \%$ à $300 \mathrm{~K}$. Dans nos échantillons, si l'on en croit l'analyse faite au moyen de l'expression (6), cette contribution positive est nulle (ou du moins négligeable) au-dessus de $10 \mathrm{~K}$. Par contre le terme négatif $E T^{2}(T / \theta)^{3} J_{3}(\theta / T)$ représente en valeur absolue près de $8 \%$ de la résistivité totale à $300 \mathrm{~K}$. Dans une analyse d'ensemble au moyen de l'expression (3) les deux contributions de signe opposé, regroupées dans le terme $B T^{2}$, peuvent alors s'annuler mutuellement. Il n'est donc pas surprenant qu'avec cette expression on obtienne comme Webb une valeur de $B$ quasi nulle, ou, comme dans notre cas, une valeur négative. Toutefois, si la valeur positive de $B$ obtenue par Webb conduit, pour $T \gtrsim 300 \mathrm{~K}$, à donner à la courbe $\rho(T)$ une courbure positive contraire à celle observée expérimentalement par Abraham et Deviot [17], notre valeur négative de B paraît beaucoup trop forte (déjà celle de $E$ semble elle-même trop importante), car l'extrapolation à $T>300 \mathrm{~K}$ de nos expressions analytiques conduirait à trouver une courbure de $\rho(T)$ beaucoup plus accentuée que celle obtenue expérimentalement.

Enfin le tableau II permet de constater, et cela indépendamment de l'expression choisie, que la part du terme $D(T / \theta)^{5} J_{5}(\theta / T)$ décroît au profit de celle du 


\begin{tabular}{|c|c|c|c|c|c|}
\hline & & TABLEAU & & & \\
\hline & $\mathrm{N}$ & b1 & $\mathrm{N}$ & & Webb [8] \\
\hline$\rho(9,5)(\mu \Omega . \mathrm{cm})$ & 0,1 & 53 & $6,63 \times$ & $10^{-2}$ & $\simeq 5 \times 10^{-3}$ \\
\hline$\rho_{0}(\mu \Omega . \mathrm{cm})$ & $\begin{array}{r}0, \\
\text { (par extr }\end{array}$ & $\begin{array}{l}50 \\
\text { apolation) }\end{array}$ & $\begin{array}{r}6,20 \times \\
(\text { par } \text { extra }\end{array}$ & $\begin{array}{l}10^{-2} \\
\text { polation) }\end{array}$ & $\simeq 8,5 \times 10^{-4}$ \\
\hline $\begin{array}{l}\text { paramètres calculés } \\
\text { au moyen de l'ex- } \\
\text { pression }\end{array}$ & 3 & 6 & 3 & 6 & 3 \\
\hline$A(\mu \Omega . \mathrm{cm})$ & 0,153 & 0,152 & $6,35 \times 10^{-2}$ & $6,22 \times 10^{-2}$ & $7,18 \times 10^{-4}$ \\
\hline$B\left(\mu \Omega . \mathrm{cm} \cdot \mathrm{K}^{-2}\right)$ & $-2,33 \times 10^{-5}$ & 0 & $-2,23 \times 10^{-5}$ & 0 & $1,11 \times 10^{-7}$ \\
\hline$C(\mu \Omega . \mathrm{cm})$ & 32,38 & 24,80 & 28,03 & 21,02 & 12,26 \\
\hline$D(\mu \Omega . \mathrm{cm})$ & 7,127 & 15,55 & 14,68 & 22,30 & 30,86 \\
\hline$E\left(\mu \Omega . \mathrm{cm} . \mathrm{K}^{-2}\right)$ & 0 & $-2,61 \times 10^{-5}$ & 0 & $-2,50 \times 10^{-5}$ & 0 \\
\hline$\theta(\mathrm{K})$ & 306,5 & 295 & 303 & 292 & 270,5 \\
\hline$\sigma(\%)$ & 1,17 & 1,16 & 0,86 & 0,83 & \\
\hline écart maximum ( $\%)$ & 3,6 & 3,25 & 3,6 & 3,1 & \\
\hline$c\left(\mu \Omega \cdot \mathrm{cm} \cdot \mathrm{K}^{-3}\right)$ & $8,11 \times 10^{-6}$ & $6,93 \times 10^{-6}$ & $7,27 \times 10^{-6}$ & $6,08 \times 10^{-6}$ & $4,54 \times 10^{-6}$ \\
\hline$d\left(\mu \Omega \cdot \mathrm{cm} \cdot \mathrm{K}^{-5}\right)$ & $3,26 \times 10^{-10}$ & $8,61 \times 10^{-10}$ & $7,15 \times 10^{-10}$ & $1,30 \times 10^{-9}$ & $2,69 \times 10^{-10}$ \\
\hline$e\left(\mu \Omega \cdot \mathrm{cm} \cdot \mathrm{K}^{-5}\right)$ & 0 & $-7,28 \times 10^{-12}$ & 0 & $-7,22 \times 10^{-12}$ & 0 \\
\hline$\frac{B T^{2}}{\rho}$ à $300 \mathrm{~K}(\%)$ & -14 & 0 & $-13,5$ & 0 & 0,1 \\
\hline $\begin{aligned} C\left(\frac{T}{\theta}\right)^{3} J_{3}\left(\frac{\theta}{T}\right) / \rho \\
\text { à } 300 \mathrm{~K}(\%)\end{aligned}$ & 102 & 82 & 90 & 70 & 44,5 \\
\hline $\begin{aligned} D\left(\frac{T}{\theta}\right)^{5} J_{5}\left(\frac{\theta}{T}\right) / \rho \\
\text { à } 300 \mathrm{~K}(\%)\end{aligned}$ & 11 & 25 & 23 & 37 & 55,4 \\
\hline $\begin{array}{c}E T^{2}\left(\frac{T}{\theta}\right)^{3} J_{3}\left(\frac{\theta}{T}\right) / \rho \\
\text { à } 300 \mathrm{~K}(\%)\end{array}$ & 0 & -8 & 0 & $-7,5$ & 0 \\
\hline
\end{tabular}

terme $C(T / \theta)^{3} J_{3}(\theta / T)$ lorsque le rapport de résistivité (c'est-à-dire la pureté) diminue. Ce résultat va être confirmé par le fait que le premier de ces termes disparaît complètement dans les alliages $\mathrm{Nb}-\mathrm{Hf}$.

4.2.2 Alliages niobium-hafnium. - Pour la majorité de ceux-ci c'est l'expression

$$
\rho=A+B T^{2}+C\left(\frac{T}{\theta}\right)^{3} J_{3}\left(\frac{\theta}{T}\right)
$$

qui représente le mieux la résistivité, avec $B$ négatif comme précédemment. Toutefois, pour quelques-uns des alliages les moins concentrés, l'expression

$\rho=A+C\left(\frac{T}{\theta}\right)^{3} J_{3}\left(\frac{\theta}{T}\right)+E T^{2}\left(\frac{T}{\theta}\right)^{3} J_{3}\left(\frac{\theta}{T}\right)$

(avec $E$ négatif) donne des résultats équivalents. Par souci d'homogénéité des résultats, nous ne présentons que ceux donnés par la relation (9). Ils sont reportés dans le tableau I avec les autres données expérimentales. Les courbes de la figure 4 qui accompagnent les points expérimentaux ont été calculées au moyen de l'expres$\operatorname{sion}(9)$.

On peut, au sujet de cette expression, faire ici les mêmes remarques qu'à propos des expressions (3) et (6) données pour le niobium. Au-dessous d'environ $20 \mathrm{~K}$, l'expression (9) se réduit à $\rho-A=B T^{2}+c T^{3}$, quantité qui, par suite que $B$ est négatif, varie plus vite que $T^{3}$, alors que nous avions assimilé la partie basse température des résultats de la figure 5 à une courbe en $T^{2,7}$. Mais nous avions pour cela pris en compte des points à $T>20 \mathrm{~K}$, où $\rho(T)-\rho_{0}$ varie moins vite que $T^{3}$. De plus, au-dessous de $20 \mathrm{~K}$, ces valeurs comportent une incertitude qui est de l'ordre des différences observées entre $A$ et $\rho_{0}$.

Nous avons tracé sur la figure 8 les valeurs de $\theta$ en 


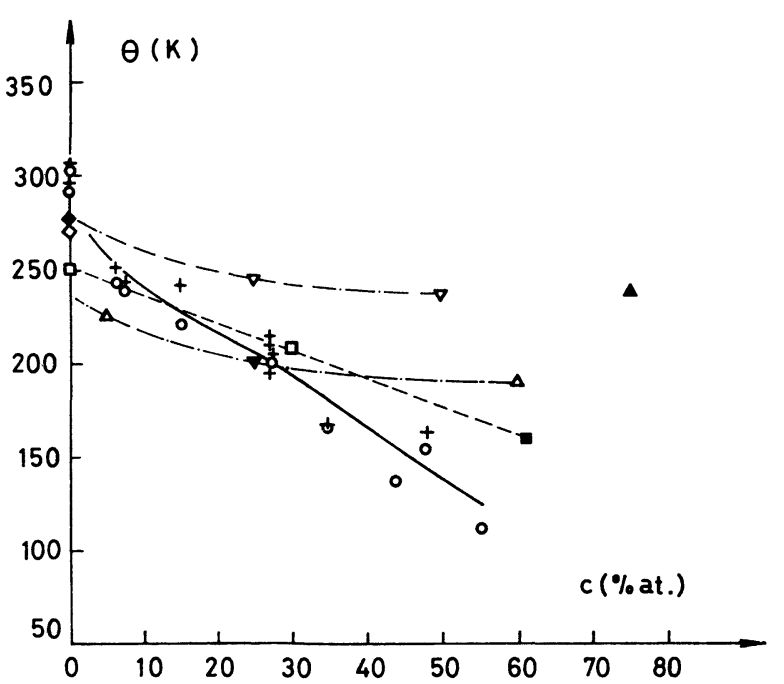

Fig. 8. - Variation de $\theta$ avec la concentration. Trait plein : nos échantillons (+ écrouis, $\bigcirc$ recuits), $\diamond$ résultat de Webb [8] pour le niobium. Les autres symboles représentent les valeurs obtenues par mesure de chaleur spécifique à basse température : niobium $[14,15,29,30]$. —...- Alliages $\mathrm{Nb}-\mathrm{Zr}$ ( $\nabla$ Heiniger et al. [14], $\nabla$ Bindari et Litvak [32], $\triangle$ Morin et Maita [33]). A Alliage Nb-Ti (Sukzrevskii et al. [34]).

Ta et alliages Ta-Hf ( $\square$ Heiniger et al. [14], $\square$ Dammer et Mulder [35]).

fonction de la concentration $c$; la chaleur spécifique des alliages $\mathrm{Nb}-\mathrm{Hf} \mathrm{n}$ 'a, semble-t-il, pas encore été mesurée à basse tempérautre, mais on peut comparer ces valeurs à celles qui ont été mesurées sur des alliages voisins : Nb-Zr [14, 32, 33], Nb-Ti [34], Ta-Hf [29, 35]. Malgré la dispersion de nos résultats et les divergences qui existent dans ceux relatifs à $\mathrm{Nb}-\mathrm{Zr}$, on peut constater que dans tous les cas, $\theta$ décroît quand la concentration croît. Cependant, la variation que nous observons est beaucoup plus importante que celle obtenue dans les autres alliages au moyen de la chaleur spécifique, et nos valeurs de $\theta$ n'ont vraisemblablement plus le bon ordre de grandeur pour les concentrations supérieures à $30 \%$. Cela peut résulter en partie du fait que la part de la résistivité qui varie avec $T$ diminue quand $c$ augmente et que par suite les paramètres $B, C$ et $\theta$ sont déterminés avec de moins en moins de précision.

La figure 9 représente la variation, en fonction de $c$, pour $T=300 \mathrm{~K}$, des termes $B T^{2}$ et $C(T / \theta)^{3} J_{3}(\theta / T)$ et de leur somme algébrique (équivalente à $\left.\rho(300)-\rho_{0}\right)$. La valeur absolue de $B T^{2}$ représente en moyenne $13 \%$ de cette somme, ce qui, comme dans le niobium, paraît exagéré et donnerait, pour $T>300 \mathrm{~K}$, à $\rho(T)$ calculé, une concavité trop accentuée.

Le paramètre $C$ est une fonction décroissante de la concentration (Fig. 10). Ce résultat donne une indication sur la variation de la constante d'interaction électron-phonon s-d, laquelle est contenue au carré dans $C$. En effet, par analogie avec l'expression de $D$, qui, d'après Ziman [23] s'écrit :

$$
D=\frac{K Q^{6} \mathrm{C}_{\mathrm{ss}}^{2}}{M \theta k_{\mathrm{F}}^{4} v_{\mathrm{s}}^{2}}
$$

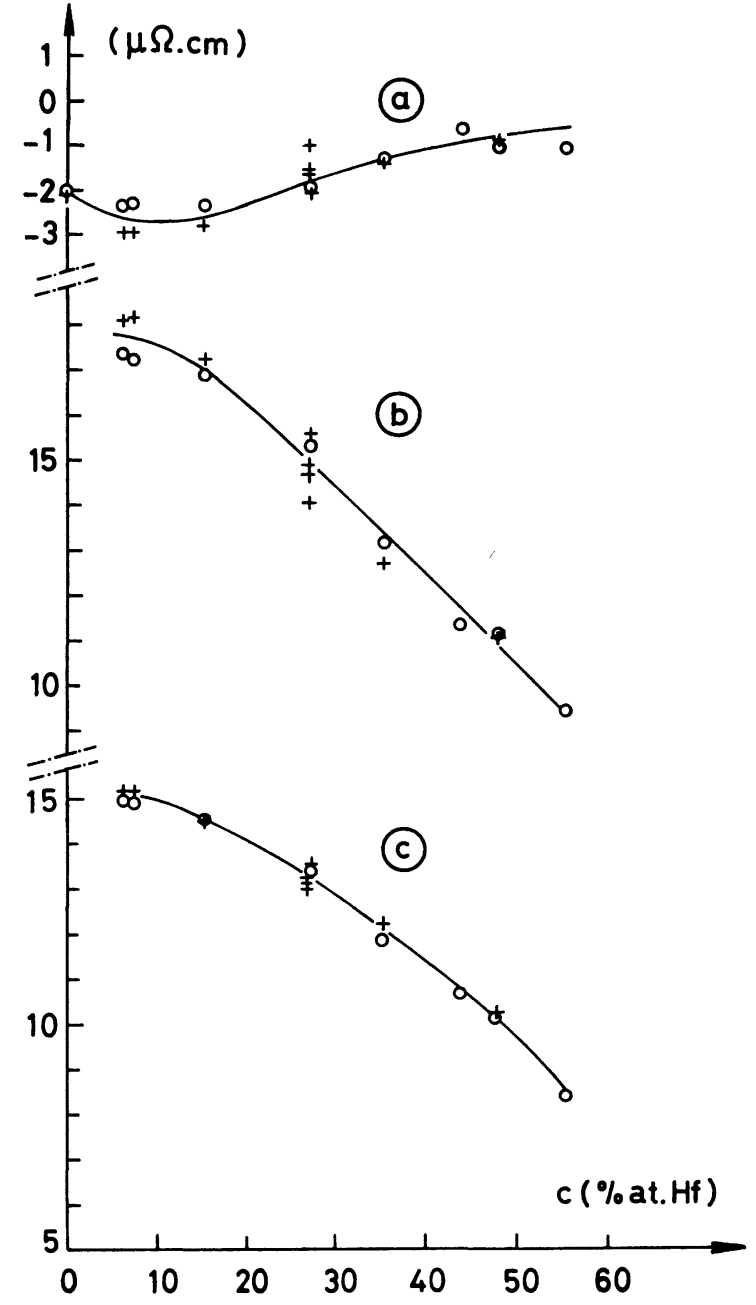

FIG. 9. - Variation avec la concentration, pour $T=300 \mathrm{~K}$, des termes $B T^{2}$ (courbe $a$ ) $C(T / \theta)^{3} J_{3}(\theta / T)$ (courbe $b$ ) et de leur somme (courbe $c$ ) dans nos échantillons ( + écrouis, $\bigcirc$ recuits).

nous pouvons mettre $C$ sous la forme

$$
C=\frac{3 K Q^{4} \mathcal{C}_{\mathrm{sd}}^{2}}{M \theta k_{\mathrm{F}}^{2} v_{\mathrm{s}} v_{\mathrm{d}}}
$$

où $\mathrm{C}_{\mathrm{ss}}$ et $\mathrm{C}_{\mathrm{sd}}$ sont des constantes d'interaction électronphonon, $Q$ le rayon de Debye, $k_{\mathbf{F}}$ le rayon de Fermi (que nous avons pris égal pour les surfaces $\mathrm{s}$ et $\mathrm{d}$ ), $v_{\mathrm{s}}$ et $v_{\mathrm{d}}$ les vitesses de Fermi s et d, $M$ la masse du volume unitaire, et $K$ un facteur numérique dans lequel nous avons regroupé un certain nombre de constantes. On ne peut prétendre calculer $\mathcal{C}_{\text {sd }}$ à partir de $C$, en raison du trop grand nombre d'approximations faites pour aboutir à la formule (11); d'ailleurs $k_{\mathrm{F}}, v_{\mathrm{s}}$ et $v_{\mathrm{d}}$ ne sont pas connus. Mais on peut supposer, qu'ainsi que $Q$, ils varient peu avec la concentration; en effet $Q$ dépend du paramètre de maille qui croît lentement [10]. Cela revient à dire que $\mathrm{C}_{\text {sd }}$ est sensiblement proportionnel à $\sqrt{C \theta M}$, quantité qui, de même que $C$ et $\theta$ varie à l'inverse de la concentration (Fig. 10). Cette variation demanderait à être confirmée par d'autres résultats. Il serait intéressant, en particulier, de la comparer à la variation du paramètre d'interaction électron-phonon 

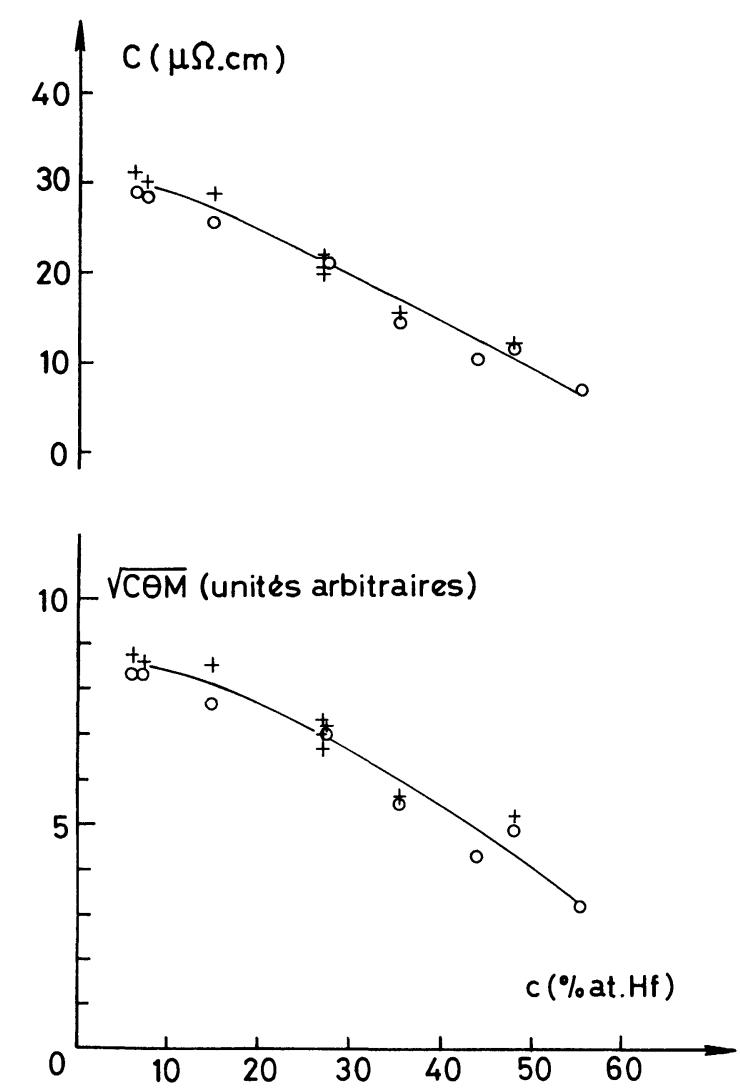

FIG. 10. - Variation du paramètre $C$ (en haut) et de la quantité $\sqrt{C \theta M}$ (en bas) dans nos échantillons (mêmes symboles que sur la figure 9).

que l'on pourrait déduire (au moyen de la relation de B. C. S., ou de celle de Mac-Millan) de mesures de chaleur spécifique à basse température.

4.3 ECARTS A La RÈGle De Matthiessen. - Les courbes $\rho_{\mathrm{i}}(T)=\rho(T)-\rho_{0}$ du niobium tracées sur la figure 3 présentent entre elles des différences trop importantes pour être attribuées à autre chose (comme par exemple à des déviations systématiques provenant de ce que ces mesures ont été effectuées au moyen de dispositifs expérimentaux différents) qu'à des écarts à la règle de Matthiessen. Nous avons calculé ces écarts point par point en retranchant aux valeurs de $\rho_{\mathrm{i}}(T)$ données par White et Woods [13], et à celles de nos échantillons, la valeur de $\rho_{\mathrm{i}}(T)$ du niobium de Webb, calculée, pour chaque température considérée, au moyen de la relation (3). Ils sont portés en coordonnées ordinaires sur la figure 11 et logarithmiques sur la figure 12 ; dans le cas de nos échantillons le résultat est identique si on le détermine par la différence des courbes calculées. A basse température il oscille autour des courbes moyennes tracées sur la figure 12, si bien qu'il est difficile de lui attribuer une loi de variation simple; dans leur plus grande partie ces courbes moyennes sont approximativement en $T^{1,5}$. Ces écarts passent par un minimum d'autant plus élevé que le rapport de résistivité du métal est plus faible. La normalisation effectuée impose qu'ils soient nuls à $295 \mathrm{~K}$,

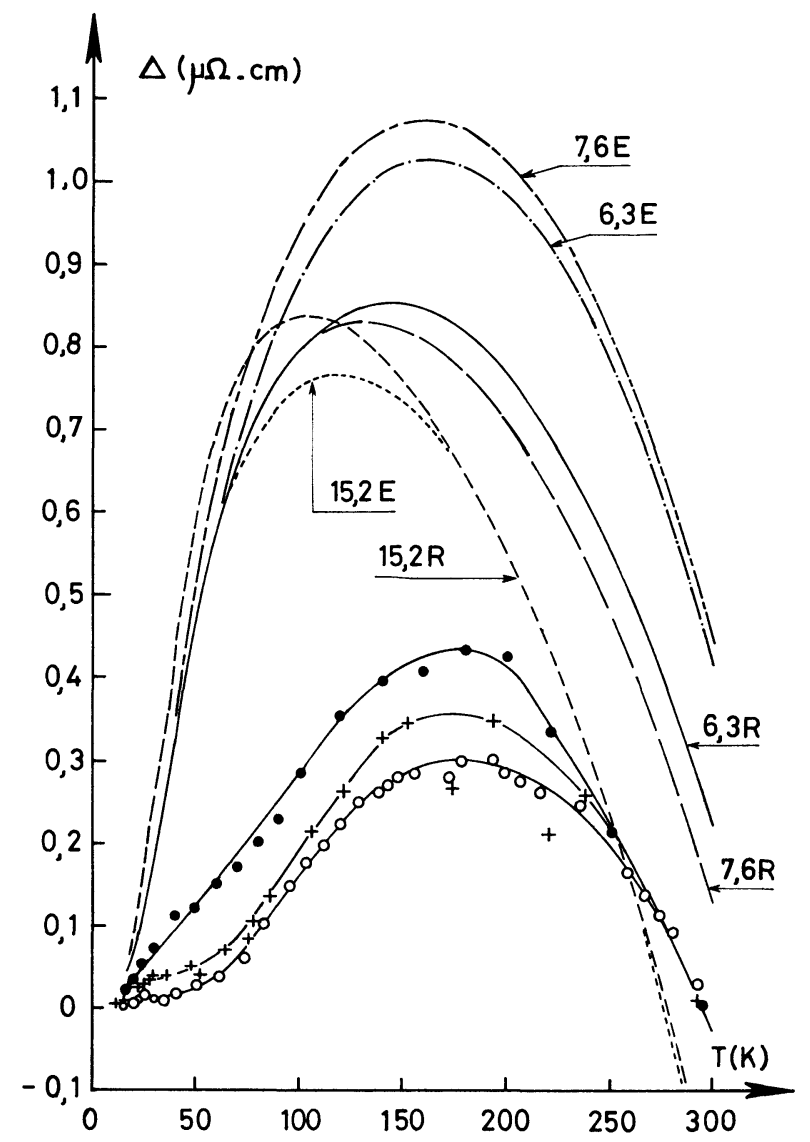

Fig. 11. - Ecarts à la règle de Matthiessen dans la résistivité du niobium et des alliages $\mathrm{Nb}-\mathrm{Hf}$.

$$
\left(\Delta=\left(\rho-\rho_{0}\right)_{\text {alliage }}-\left(\rho-\rho_{0}\right)_{\text {pur }}\right)
$$

en prenant pour $\left(\rho-\rho_{0}\right)_{\text {pur }}$ les résultats de Webb [8]. calculé à partir des résultats de White et Woods [13]. + Nb1, O Nb2. Les autres courbes sont relatives aux échantillons $\mathrm{Nb}$-Hf pour les concentrations et l'état métallurgique indiqués.

ce qui est sans doute peu réaliste, mais un autre choix affecterait assez peu les résultats à basse température. Nous avons calculé également, pour $T<47 \mathrm{~K}$, l'écart existant entre la résistivité $\rho_{\mathrm{i}}(T)$ donnée par Clinard et Kempter [16] et celle du niobium de Webb ; il est très proche de celui de l'échantillon $\mathrm{Nb} 1$. Il existe aussi un écart entre le résultat de Webb et les valeurs données par Abraham et Deviot [17], mais ces derniers n'ayant pas déterminé leur résistivité résiduelle, cet écart n'est pas chiffrable à basse température.

Nous avons déterminé également l'écart $\Delta$ entre la résistivité des alliages $\mathrm{Nb}-\mathrm{Hf}$ et celle du niobium de Webb, à partir des relations numériques représentatives de la résistivité de ces échantillons. Les courbes d'écart relatives aux alliages à $6,3 \%, 7,6 \%$ et $15,2 \%$ sont tracées sur les figures 11 et 12 en compagnie de celles du niobium ; les autres sont représentées sur les figures 13 et 14 . Les concentrations de ces alliages sont telles que l'on ne peut plus à proprement parler désigner ces courbes par l'expression " écarts à la règle de Matthiessen ), qui est généralement réservée à des alliages dilués. On voit pourtant une continuité depuis 


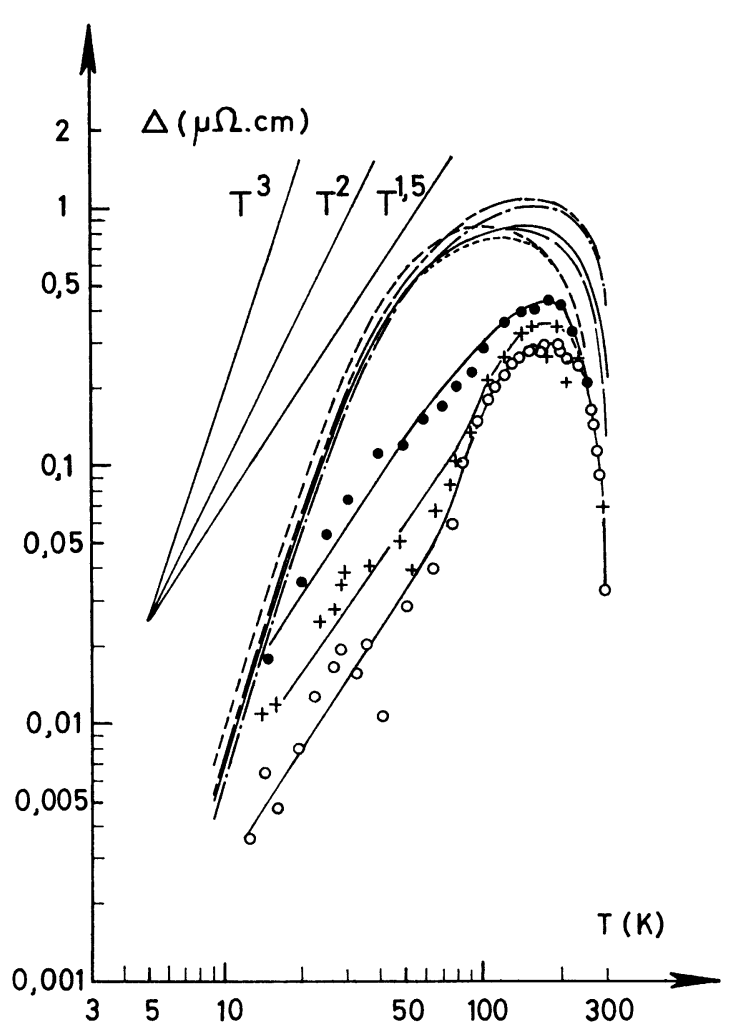

FIG. 12. - Résultats de la figure 11, représentés en coordonnées logarithmiques afin de détailler la variation à basse température.

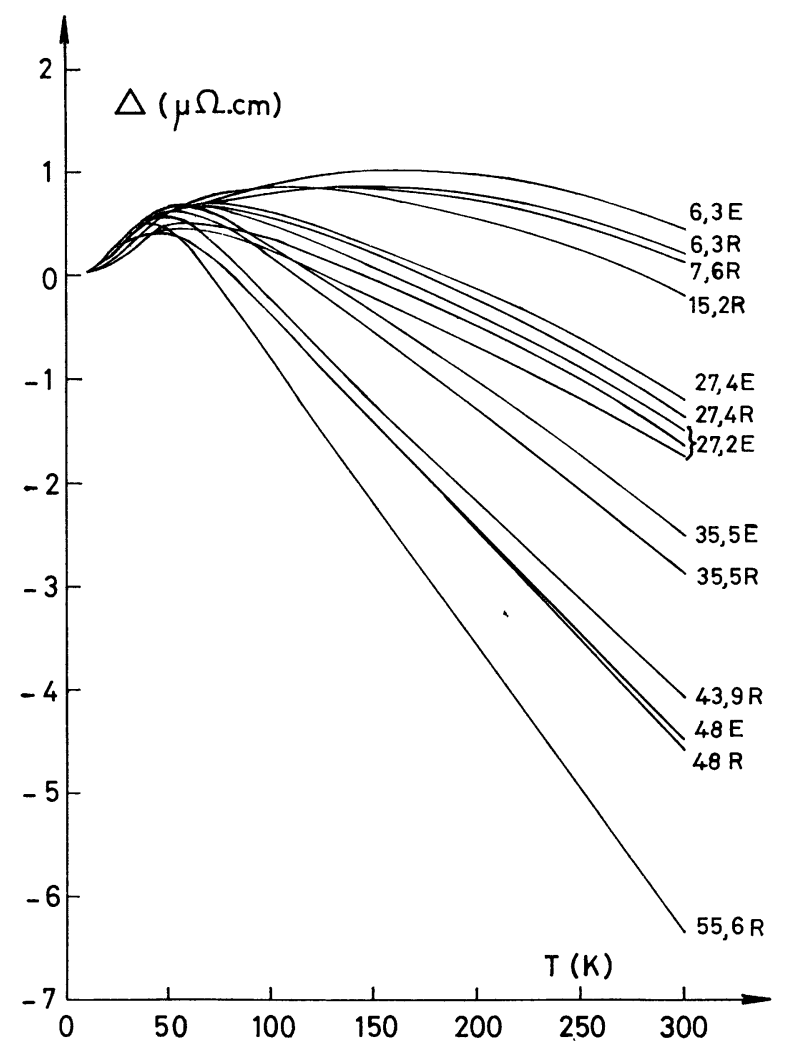

FIG. 13. - Ecarts de la résistivité des alliages Nb-Hf, par rapport au niobium (mêmes définitions que pour les courbes de la figure 11).

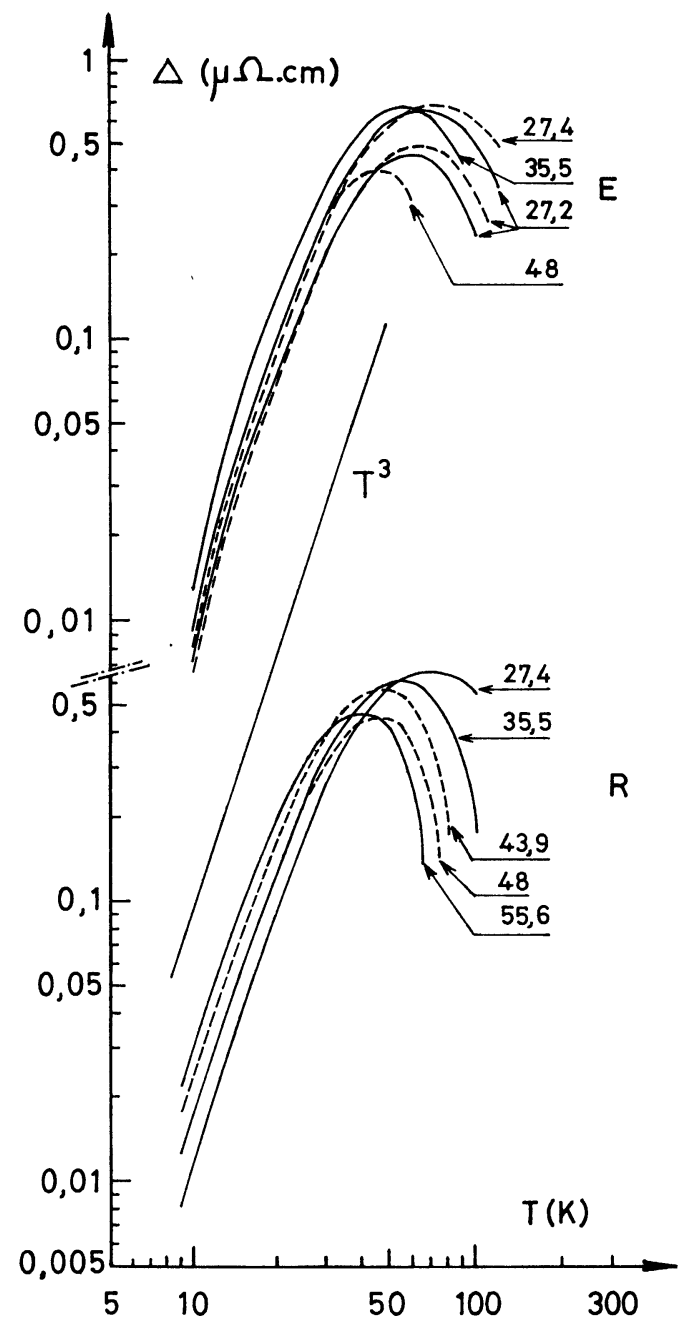

Fig. 14. - Partie basse température des résultats de la figure 13, en coordonnées logarithmiques. En haut échantillons écrouis. En bas, échantillons recuits.

le niobium jusqu'à l'alliage le plus concentré en hafnium. De plus on reconnaît sur la figure 13 une forme générale de courbe qu'il n'est pas rare de rencontrer dans l'étude des écarts à la règle de Matthiessen. Dans la revue qu'il en a faite, Bass [36] ne cite pas d'exemple d'étude d'écarts dans des alliages de niobium, mais la forme des courbes se retrouve par exemple dans les écarts des alliages dilués $\mathrm{Cd}-\mathrm{Ag}, \mathrm{Cd}-\mathrm{Mg}, \mathrm{Mg}-\mathrm{Al}$ [37] et $\mathrm{Al}-\mathrm{Ag}[37,38]$. Il apparaît toutefois un certain nombre de différences entre ces courbes et nos résultats. Dans les alliages dilués la température du maximum est au plus de l'ordre de $\theta / 5$. Sur les courbes de la figure 13 l'amplitude et la température du maximum décroissent régulièrement quand la concentration augmente; en prenant pour $\theta$ les températures de Debye que nous avons déterminées, on voit que la température de ce maximum, de l'ordre de $\theta / 1,5$ pour les faibles concentrations, s'abaisse à $\theta / 2,5$ pour les fortes concentrations. Dans les alliages Nb-Hf, comme dans les alliages dilués, l'écart à l'ambiante dépend de la concentration, mais l'ordre de grandeur est très différent, puisqu'il est proche de $-6 \mu \Omega . \mathrm{cm}$ pour l'échantillon à $55,6 \%$ 
de Hf; on voit également ici une influence de l'écrouissage. Pour les échantillons recuits, les écarts à $300 \mathrm{~K}$ sont pratiquement proportionnels à la résistivité résiduelle des échantillons. Par contre, au-dessous de 20 à $30 \mathrm{~K}$, ils dépendent très peu de la concentration et varient pratiquement en $T^{3}$. Un comportement analogue à celui-ci a été observé dans les alliages dilués cités plus haut, et, en général dans les alliages non magnétiques d'aluminium, dilués [39] ou concentrés [40]; dans ces derniers, plus précisément, l'écart est proportionnel à $T^{3} \log \rho_{0}$. De nombreux modèles théoriques ont été proposés pour interpréter ces écarts à basse température [36-41]. Il est difficile de dire lequel pourrait convenir dans le cas des alliages de niobium ; on peut simplement constater une analogie entre l'analyse que nous venons de faire et le modèle de Campbell, Caplin et Rizzuto [41], qui propose de décrire la résistivité des alliages d'aluminium par une expression de la forme

$$
\left(\frac{T}{\theta}\right)^{3} \int_{0}^{\theta / T} z^{2}\left(e^{z}-1\right)^{-1} \mathrm{~d} z,
$$

alors que le métal pur est en $T^{5}$. Toutefois, cette intégrale, établie à partir de considérations physiques autres que celles employées par Wilson pour aboutir à l'expression (2), est différente de $J_{3}(\theta / T)$ que nous utilisons ici.

5. Conclusion. - Notre analyse de la résistivité électrique du niobium entre $T_{\mathrm{c}}$ et l'ambiante, a donné des températures de Debye en bon accord avec celles que fournissent des mesures de chaleur spécifique à basse température. Cela confirme que, si précédemment, dans le cas des métaux de transition, on obtenait, à partir de la résistivité, des températures de Debye très différentes de celles déduites des mesures de chaleur spécifique (voir par exemple les valeurs données par Meaden [24]) c'était principalement parce que $\theta$ était déterminé en appliquant à la résistivité une loi qui contenait uniquement le terme en $J_{5}$. Dans le cas des alliages $\mathrm{Nb}-\mathrm{Hf}$, nous n'avons pu comparer nos températures de Debye qu'à celles d'autres systèmes, mais cela a montré que, pour les concentrations en hafnium inférieures à $30 \%$ at., les valeurs de $\theta$ que nous avons obtenues sont relativement correctes. Par contre, à partir du paramètre $C$ on ne peut obtenir qu'une information très qualitative sur la constante d'interaction électron-phonon, à savoir que celle-ci est, à l'image de $C$, une fonction décroissante de la concentration.
Nos calculs montrent essentiellement que dans la résistivité électrique du niobium, la part du terme $D(T / \theta)^{5} J_{5}(\theta / T)$ diminue avec la pureté, au profit du terme $C(T / \theta)^{3} J_{3}(\theta / T)$, pour disparaître complètement dans les alliages Nb-Hf. Ainsi, en négligeant les termes de faible valeur $B T^{2}$ ou $E T^{2}(T / \theta)^{3} J_{3}(\theta / T)$, l'expression mathématique des écarts à la règle de Matthiessen dans les alliages dilués de niobium, peut s'écrire, en supposant $\theta$ invariant :

$$
\begin{aligned}
\Delta(T)= & {\left[\rho(T)-\rho_{0}\right]_{\mathrm{a}}-\left[\rho(T)-\rho_{0}\right]_{\mathrm{p}} } \\
\simeq\left(C_{\mathrm{a}}-C_{\mathrm{p}}\right)\left(\frac{T}{\theta}\right)^{3} J_{3}\left(\frac{\theta}{T}\right)+ & \\
& \quad+\left(D_{\mathrm{a}}-D_{\mathrm{p}}\right)\left(\frac{T}{\theta}\right)^{5} J_{5}\left(\frac{\theta}{T}\right)
\end{aligned}
$$

les indices a et $\mathrm{p}$ étant relatifs respectivement à l'alliage et au métal pur. Dans les alliages plus concentrés, où $\theta_{\mathbf{a}}$ est différent de $\theta_{\mathrm{p}}$, cet écart devient

$$
\begin{aligned}
\Delta(T) \simeq C_{\mathrm{a}}\left(\frac{T}{\theta_{\mathrm{a}}}\right)^{3} J_{3}\left(\frac{\theta_{\mathrm{a}}}{T}\right) \\
-\left[C_{\mathrm{p}}\left(\frac{T}{\theta_{\mathrm{p}}}\right)^{3} J_{3}\left(\frac{\theta_{\mathrm{p}}}{T}\right)+D_{\mathrm{p}}\left(\frac{T}{\theta_{\mathrm{p}}}\right)^{5} J_{5}\left(\frac{\theta_{\mathrm{p}}}{T}\right)\right] .
\end{aligned}
$$

Ces expressions donnent une variation approximativement en $T^{3}$ à basse température, en raison de la prépondérance des termes en $T^{3} J_{3}$; cela ne s'est apparemment pas vérifié pour les écarts dans le niobium, car nous ne disposions pas dans ce cas de mesures, et en particulier d'une définition de $\rho_{0}$, suffisamment précises, mais décrit correctement les écarts dans les alliages $\mathrm{Nb}-\mathrm{Hf}$.

Remerciements. - L'un de nous (J. T.) remercie l'Instituto de Alta Cultura et l'Instituto de Física et Matemática de Lisbonne pour l'appui qu'ils lui ont accordé ainsi que le C. R. T. B. T. de Grenoble pour l'accueil qui lui a été réservé et l'aide qui lui a été apportée au cours de ce travail.

Nous remercions en particulier O. Béthoux dont les connaissances sur les propriétés métallurgiques et supraconductrices des alliages $\mathrm{Nb}-\mathrm{Hf}$ nous furent précieuses, $\mathrm{P}$. Groh pour l'intérêt qu'il a pris à la réalisation de l'appareillage, D. Brochier qui nous a fait profiter de son expérience en programmation, ainsi que R. Tur pour sa participation au travail expérimental.

\section{Bibliographie}

[1] Ho, J. C., Collings, E. W., Phys. Rev. B 6 (1972) 3727.

[2] Hulm, J. K., Blaugher, R. D., Phys. Rev. 123 (1961) 1569.

[3] Berlincourt, T. G., Hake, R. R., Phys. Rev. 131 (1963) 140.

[4] Siemens, R. E., Oden, L. L., Deardorff, D. K., U. S. Bureau of Mines (1969) Report of investigations 7258.

[5] GuÉRIN, J. Y., Thèse docteur-ingénieur, Grenoble (1970) non publiée.

[6] Bethoux, O., Guérin, J. Y., Proc. 12th Int. Conf. Cryo. Low
Temp. Phys., Kyoto (1970) (Academic Press of Japan) 1971, Section B, 379.

[7] Koch, C. C., Carpenter, R. W., Phil. Mag. 25 (1972) 303.

[8] Webs, G. W., Phys. Rev. 181 (1969) 1127.

[9] Siemens, R. E., Babitzke, H. R., Kato, H., U. S. Bureau of Mines (1964) Report of Inv. 6492.

[10] TAYlor, A., Doyle, N. J., J. Less-Common Metals 7 (1964) 37. 
[11] Tylkina, M. A., Tsyganova, I. A., Savitskit, E. M., $Z h$. Neorgan. Khim. 9 (1964) 1650 (traduction anglaise : Russian, J., Inorg. Chem. 9 (1964) 893).

[12] Carpenter, R. W., Liu, C. T., Mardon, P. G., Metall. Trans. 2 (1971) 125.

[13] White, G. K., Woods, S. B., Phil. Trans. Roy. Soc. (London) A 251 (1959) 273.

[14] Heiniger, F., Bucher, E., Muller, J., Phys. Mat. Cond. 5 (1966) 243.

[15] Ferreira da Silva, J., Burgemeister, E. A., Dokoupil, Z., Physica 41 (1969) 409.

[16] Clinard, F. W., Jr, Kempter, C. P., J. Less-Common Metals 15 (1968) 59.

[17] Abraham, J. M., Deviot, B., J. Less-Common Metals 29 (1972) 311 .

[18] Ames, S. L., McQuillan, A. D., Acta Met. 2 (1954) 831.

[19] Evans, D. J., Erickson, R. A., J. Appl. Phys. 36 (1965) 3517.

[20] Colling, D. A., Ralls, K. M., Wulff, J., Trans. Met. Soc. A. I. M. E. 236 (1966) 1218.

[21] Cometto, D. J., Houze G. L., Jr, Hehemann, R. F., Trans. Met. Soc. A. I. M. E. 233 (1965) 30.

[22] Brotzen, F. R., Harmon, E. L., Jr, Troiano, A. R., Trans. A. I. M.E. 203 (1955) 413.

[23] Ziman, J. M. dans Electrons and Phonons (Clarendon Press, Oxford) 1960, chap. VII.

[24] Meaden, J. T., dans Electrical Resistance of Metals (Plenum Press, New York) 1965.

[25] Wilson, A. H., Proc. R. Soc. (London) A 167 (1938) 580.

[26] Mattheiss, L. F., Phys. Rev. B 1 (1970) 373.
[27] Cezairliyan, A., J. Res. Nat. Bur. Stand., ser. A 75 (1971) 565.

[28] Motr, N. F., Jones, H., The theory of the Properties of Metals and Alloys (Clarendon Press, Oxford) 1936, (Dover Publ., New York) 1958.

[29] Kimura, Y., Ohtsuka, T., Matsui, T., Mizusaki, T., Phys. Lett. 29A (1969) 284.

[30] Moore, M. A., Paul, D. I., Solid State Commun. 9 (1971) 1303.

[31] Linkoaho, M., Phil. Mag. 23 (1971) 191.

[32] Bindari, A. E., Litvak, M. M., J. Appl. Phys. 34 (1963) 2913.

[33] Morin, F. J., Maita, J. P., Phys. Rev. 34 (1963) 2913.

[34] SukharevskiI, B. Ya., Alapina, A. V., Duschechkin, Yu. A., Zh. Eksp. Teor. Fiz. 54 (1968) 1675 (traduction anglaise : Sov. Phys. JETP 27 (1968) 897).

[35] Dammer, G., Mulder, K., cités par Gey, W., Köhnlein, D., Z. Phys. 255 (1972) 308.

[36] Bass, J., Adv. Phys. 21 (1972) 431.

[37] Seth, R. S., Woods, S. B., Phys. Rev. B 2 (1970) 2961.

[38] Panova, G. Kh., Zhernov, A. P., Kutaitsev, V. I., $\boldsymbol{Z h}$. Eksp. Teor. Fiz. 56 (1969) 104 (traduction anglaise : Sov. Phys. JETP 29 (1969) 59).

[39] Caplin, A. D., Rizzuto, C., Australian J. Phys. 24 (1971) 309.

[40] Krsnik, R., Babić, E., Rizzuto, C., Solid State Commun. 12 (1973) 891.

[41] Campbell, I. A., Caplin, A. D., Rizzuto, C., Phys. Rev. Lett. 26 (1971) 239. 